$A D$

Award Number: W81XWH-11-1-0402

TITLE: Mammary Cancer and Activation of Transposable Elements

PRINCIPAL INVESTIGATOR: Dr. John Edwards

CONTRACTING ORGANIZATION: Washington University

Saint Louis, MO 63130-4862

REPORT DATE: March 2015

TYPE OF REPORT: Final

PREPARED FOR: U.S. Army Medical Research and Materiel Command Fort Detrick, Maryland 21702-5012

DISTRIBUTION STATEMENT: Approved for Public Release; Distribution Unlimited

The views, opinions and/or findings contained in this report are those of the author(s) and should not be construed as an official Department of the Army position, policy or decision unless so designated by other documentation. 


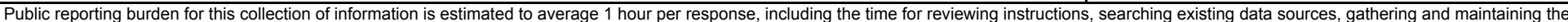

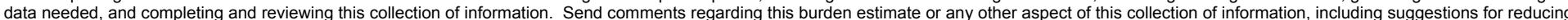

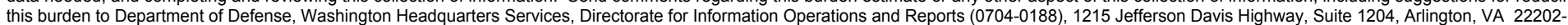

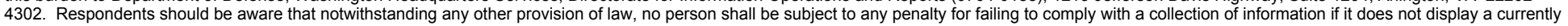
valid OMB control number. PLEASE DO NOT RETURN YOUR FORM TO THE ABOVE ADDRESS

\begin{tabular}{l|l} 
1. REPORT DATE & 2. REPORT TYPE \\
March 2015 & Final \\
\hline
\end{tabular}

March 2015

4. TITLE AND SUBTITLE

Mammary Cancer and Activation of Transposable Elements
3. DATES COVERED

01 Sep $2011-31$ Dec 2014

5a. CONTRACT NUMBER

W81XWH-11-1-0402

5b. GRANT NUMBER

5c. PROGRAM ELEMENT NUMBER

5d. PROJECT NUMBER

5e. TASK NUMBER

5f. WORK UNIT NUMBER

E-Mail: jedwards@dom.wustl.edu

7. PERFORMING ORGANIZATION NAME(S) AND ADDRESS(ES)

Washington University

Saint Louis, MO 63130

6. AUTHOR(S)

John R. Edwards, PhD

8. PERFORMING ORGANIZATION REPORT NUMBER
9. SPONSORING / MONITORING AGENCY NAME(S) AND ADDRESS(ES)

U.S. Army Medical Research and Materiel Command

Fort Detrick, Maryland 21702-5012
10. SPONSOR/MONITOR'S ACRONYM(S)

11. SPONSOR/MONITOR'S REPORT NUMBER(S)

12. DISTRIBUTION / AVAILABILITY STATEMENT

Approved for Public Release; Distribution Unlimited

\section{SUPPLEMENTARY NOTES}

\section{ABSTRACT}

The purpose of this project is to investigate molecular events occurring in the preclinical stages of mammary cancer. Specifically, the project investigates the intersection between the development of genome demethylation, retrotransposon transcriptional activity, and retrotransposon-driven transcription of cellular genes in an engineered mouse model of mammary cancer. RNA-seq and Methyl-MAPS methylation analysis was performed on uni-parous and tri-parous tumor-prone and control mice. We discovered genome-wide demethylation that occurs in tumor-prone tri-parous mice, that differs substantially from the long-hypomethylated domains that characterize breast tumors. We thus propose that the wide-spread remodeling of DNA methylation patterns found in tumors may begin at this early pre-clinical stages modeled in this study. However, these changes do not appear to impact transcription at this stage. During this project we have developed powerful computational tools for elucidating relationships between DNA methylation and expression. These tools have broad potential to affect our understanding of the functions of DNA methylation in breast cancer and may impact cancer prevention in the future.

\section{SUBJECT TERMS}

Breast cancer, epigenetic, DNA methylation, retrotransposon, preclinical cancer development, deep sequencing

16. SECURITY CLASSIFICATION OF:

\section{a. REPORT}

\section{b. ABSTRACT}

U
17. LIMITATION OF ABSTRACT
18. NUMBER OF PAGES

27 19a. NAME OF RESPONSIBLE PERSON USAMRMC 19b. TELEPHONE NUMBER (include area code) 
Table of Contents

Page

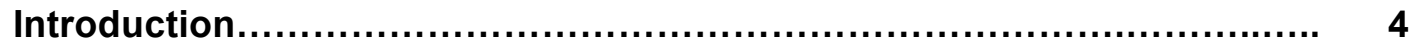

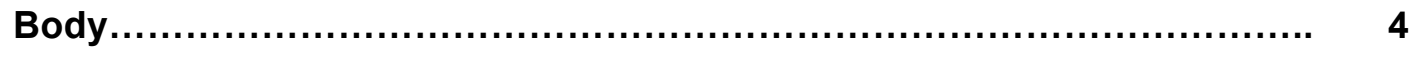

Key Research Accomplishments......................................... 11

Reportable Outcomes..................................................... 12

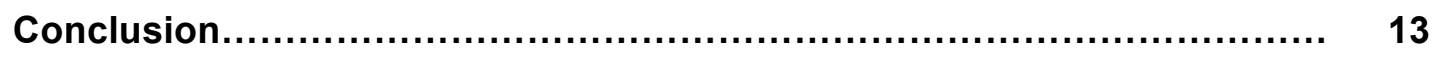

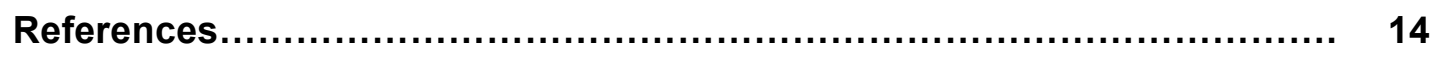

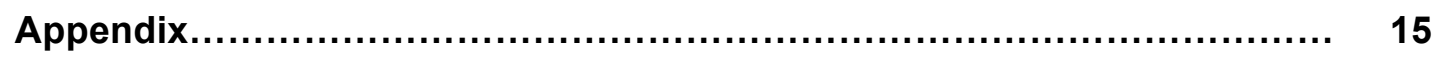




\section{Introduction}

This project is designed to address the subject of mammary cancer development. The purpose of the project is to investigate molecular events occurring in the preclinical stages of mammary cancer; the results may lead to insights into cancer prevention in the future. Specifically, the project investigates the intersection between genome demethylation, retrotransposon transcriptional activity, and retrotransposon-driven transcription of cellular genes. Retrotransposon promoters are well recognized to function as alternative promoters for different cellular genes, generating chimeric transcripts that may or may not function in the same way as transcripts from the regular gene promoter. Transcriptional activation of retrotransposons is strongly linked with their CpG DNA methylation, and global genomic demethylation is one of the commonest molecular changes in malignancies. The project tests the hypothesis that, in preclinical stages of tumor development, progressive genomic demethylation leads to increased transcriptional activity of retrotransposons and this, in turn, leads to transcription of otherwise silent genes, potentially setting up molecular conditions that favor cancer development. Our collaborator, Dr. Anne Peaston, developed a genetically engineered mouse model in which a specific mammary cell population is fluorescently marked upon initial transcriptional activation of the SV40 large T antigen (SV40Tag) oncogene. SV40Tag is transcriptionally activated during pregnancy and lactation, and the mice are predisposed to develop mammary cancer after three pregnancy and lactation cycles (triparous), but not after one cycle (uniparous). Using this model, populations of marked cells can be collected for integrated analysis of gene expression, promoter usage, and DNA methylation after defined amounts of exposure to SV40Tag during different stages of preclinical cancer development.

\section{Body}

\section{Development of New Computational Tools}

We have developed the WIMSi (Washington University Methylation Signatures) pipeline to enable us to use genome-wide methylation and expression data to examine the relationship between DNA methylation and expression. An initial description of the method and results can be found in VanderKraats et al, 2013 (a copy of the full manuscript is in the appendix).

In brief, current computational tools focus on methods to compute accurate methylation levels from the data, methods to determine differentially methylated regions, and visualization tools, such as genome browsers. These approaches have elucidated the genomic organization of these marks, but they do not sufficiently address how changes at individual loci potentially affect function. Correspondingly, such methods find only a modest negative correlation between differential DNA methylation at promoters and expression. While it is possible that DNA methylation is not a strong modifier of expression, it is more likely that our computational tools are insufficient. We hypothesize that stronger associations are not observed because existing analysis methods oversimplify their representation of the data such that they do not capture the diversity of existing methylation patterns. This includes changes at the $\mathrm{CpG}$ island (region over-represented for CG dinucleotides) at a gene's $\mathrm{TSS}$, changes at $\mathrm{CpG}$ island shores and the formation of long partially-hypomethylated domains. In addition, there are not methods to systematically search for new patterns.

We have developed a new set of tools for discovering differential methylation patterns associated with expression change using genome-wide high-resolution methylation data: we represent differential methylation as an interpolated curve, or signature, and then identify groups of genes with similarly-shaped signatures and corresponding expression changes. Our methods uncover a diverse set of DNA methylation patterns that are conserved across a variety of normal and cancerous tissues and cell lines. Our initial implementation and results are described in VanderKraats et al. 2013. Applying WIMSi to a variety of datasets, we have obtained the same basic set of methylation patterns when one compares stem cells to differentiated cells (or derived precursors), non-tumorigenic to breast tumor cells, cancer cells of different types, and different tissues. This supports the notion that these patterns are generalizable and thus that the mechanisms of how methylation and expression interact are the same across different biological contexts.

We have also applied WIMSi in two collaborative projects to demonstrate its utility. Using WIMSi, we 
discovered methylation signatures associated with cellular senescence that are similar to those found in cancer (Cruickshanks et al 2013). We further found a methylation signature associated with acute myeloid leukemia that could be reversed upon treatment with DNA methyltransferase inhibitors (Lund et al 2014). The WIMSi pipeline is publically available on SourceForge at http://sourceforge.net/projects/wimsi/.

\section{Gene List Generation}

Enumerating a list of genes for which expression and methylation changes are potentially linked is a primary interest of any genome-wide methylation profiling experiment, and is one of the direct goals of this project. The WIMSi method outlined above is tuned to discover patterns, and thus produces a conservative gene list potentially prone to false positives. Our initial publication describes one way to use this method to generate a gene list, however, we have further refined our approach using a set of supervised machine learning methods.

Our tool (DTW-kNN, Dynamic Time Warping based kNearest Neighbors) identifies which genes have associated methylation and expression changes in a pair-wise experiment (i.e. a single tumor sample compared to a normal sample). We have evaluated our tool against other methods used in the literature using whole-genome bisulfite sequencing data recently made available from the Cancer Genome Atlas (TCGA) project (Fig. 1 and Table 1). The single window classifier is a decision-tree based classifier built on the average methylation level of a single window around the gene's TSS. During training a number of parameters such a window size and number of CpGs are used. The DMR (Differentially Methylated Region) classifier uses classic published definitions for DMRs to compare against (Hansen et al 2012, VanderKraats et al. 2013), but optimizes all DMR parameters during training. The ROI, Region of Interest, classifier, is a random forest model that uses the average methylation of multiple gene regions ( $\mathrm{CpG}$ islands, transcription start site, first exons, gene body, and last exon) as inputs and is an adaptation of a recent method developed to predict absolute expression levels (Lou et al. 2014).

Our method outperforms commonly used methods in the literature in generating both more accurate and longer gene lists. Further, we conclude that the single window and DMR models commonly used in the literature are insufficient to capture the complexity of the relationships between DNA methylation and expression. From Table 1, at 95\% accuracy, we observe that DTW-kNN returns 55-606 genes depending on the tumor sample, which is much more than the next best classifier (Single Window). We also observe the ineffectiveness of the Region of Interest (ROI) classifier in the leave-one-sample-out paradigm, underscoring the importance of regional methylation shape in understanding differential expression. These results suggest that the number of loci potentially affected by DNA

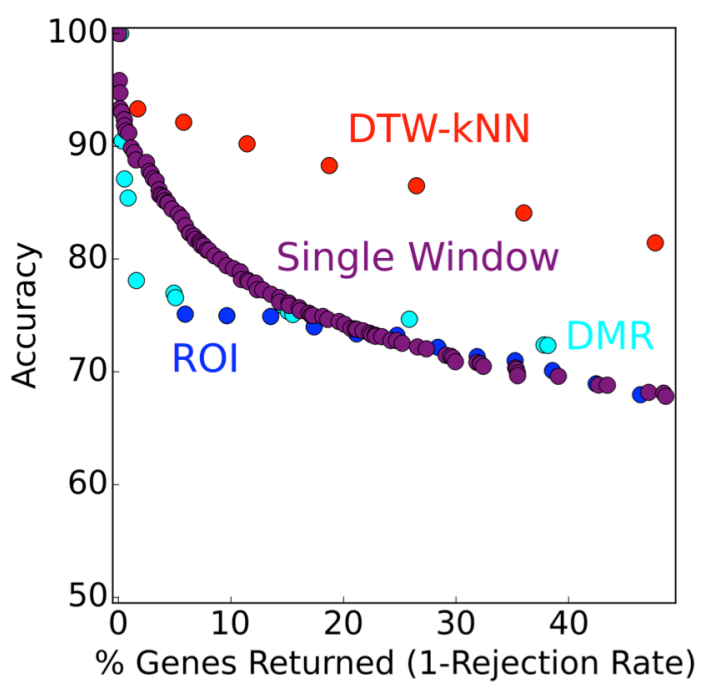

Figure 1. The average accuracy of each classifier is shown versus the average percentage of genes returned. The percentage of genes returned is equivalent to the percentage of 1-rejection rate. Addition of a rejection rate allows for the fact that for some genes methylation will not be predictive of transcriptional activity. Up and to the right defines better performance. The DTW-kNN and ROI classifiers are evaluated using leaveone-sample-out evaluation. The Single Window and DMR classifiers were purposefully trained on the testing data to demonstrate that these models cannot capture the underlying relationships between DNA methylation and expression. $50 \%$ accuracy is equivalent to a random guess.

Table 1: Range of genes returned per tumor at $95 \%$ accuracy for each classifier. No genes were identified in any sample for the ROI classifier at this accuracy level.

\begin{tabular}{ll}
\multicolumn{1}{c}{ Classifier } & Number of Genes \\
\hline DTW-kNN & $55-606$ \\
Single Window & $19-46$ \\
DMR & $4-18$ \\
ROI & -
\end{tabular}


methylation in primary breast tumors has been vastly understated. Preliminary results from this method were presented at the 2014 International Conference on Intelligent Systems for Molecular Biology.

Our findings suggest that the role of DNA methylation cannot be fully described by simply characterizing every gene as "methylated" or "unmethylated". Using our new method, we have found and described a variety of methylation patterns that correlate with expression change. The true power of this method is in its ability to discover and separate distinct patterns without a priori knowledge about existing correlations, which cannot be accomplished with contemporary approaches. This allows us to realize the full potential of unbiased genomewide profiling of DNA methylation to reveal previously unknown information about methylation's functional role. This ability will be especially important when examining regulatory elements within retroelements as will be performed in this work.

One additional implication of these results also becomes clear. The simplified models used in prior approaches at best produce weak correlations. However, if one considers a more formal description of the underlying patterns of methylation changes, methylation and expression data are highly correlated. This tool was designed to start from a list of expression data, corresponding transcription start sites (TSSs) and high-resolution genome-wide methylation data such as from Methyl-MAPS. Thus it fits perfectly into the framework of this proposal where we have obtained RNA-seq expression and Methyl-MAPS methylation data for each sample. We have further adapted our tool to address the regulation of retrotransposons promoters and we have performed some initial analyses of LTR-driven lncRNAs as a successful proof-of-concept.

\section{Expression Analysis of uni- and triparous mice}

RNA-Seq analyses using long-paired end reads were performed for two replicates. One "replicate" consists of four samples, or each combination of uni- or triparous, and control or SV40+/- mice. See Dr. Peaston's report for a complete

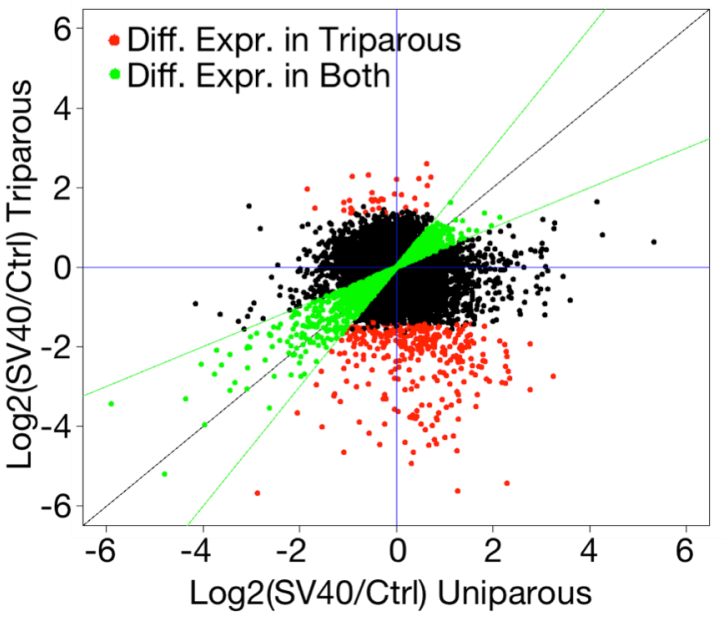

Figure 2. RNA-seq analysis of SV40+/- and control, uniparous and triparous mice. Genes differentially expressed only in SV40+/- versus control (Ctrl) triparous mice are indicated by red dots, while genes whose expression changes by similar amounts in both uni- and triparous mice are indicated in green. description of the mouse-breeding design and results. For each sample, 30-46 million paired reads were aligned to the mouse genome (mm10) using Tophat (Trapnell et al. 2009). Mapped reads were aligned to Ensembl genes using htseq and differential expression was computed using edgeR (Robinson et al. 2010). Genes that were at least 2-fold up or down regulated with and FDR (False Discovery Rate) $<0.05$ were deemed significant. To focus on genes that were differentially expressed in triparous mice, but were not up-regulated solely due to the induction of SV40 in tumor-prone mice, we removed all genes that were up- or down-regulated in uniparous mice. This left 318 genes that were uniquely down and 34 uniquely up in tumor-prone triparous mice (Fig. 2). Ontology analysis using DAVID (Huang et al. 2009) of down-regulated genes indicated that these genes were over-represented for genes involved in the cell cycle and immune response. Genes regulating cell cycle control could represent the early inactivation of checkpoints that create a permissive environment for tumor proliferation. We are currently following up on many of these genes to understand how they may be involved in setting the regulatory environment to facilitate tumor development in these mice.

Surprisingly, when we analyzed retrotransposon derived sequences we found their expression dropped in tumorprone triparous mice by an average of $\sim 18 \%$. This occurred across nearly every transposable element family. We are currently working to validate these findings. Analyses of chimeric transcripts (containing both retroelement and coding sequence) are underway. 
Methyl-MAPS analysis was performed for one experimental replicate. Methylation levels were determined for $\sim 5$ million CpG sites per sample at an average coverage level from 15-27x. We encountered difficulties during the final stages of library construction for replicate 2 . We are working to salvage these samples and perform Methyl-MAPS analysis. For replicate 3 it was determined that there was insufficient material to build sequencing libraries and thus the DNA has been held for validation of target regions identified from the other replicates.

The average methylation levels of $100 \mathrm{~kb}$ bins is shown in Fig. 2. These data indicate that methylation levels are constant in SV40+/- and control uniparous mice. However, methylation levels appear to drop $(\sim 3.5 \%)$ in tumorprone SV40+/- triparous mice versus controls. As indicated in Fig. 3 and 4, this appears to be a genome-wide phenomena and is not consistent with this decrease being caused by long-hypomethylated domains as has been recently described in several tumor types including breast (Berman et al 2012, Hon et al 2012). Methylation decreases were found in all genomic compartments including genes, promoters, and repeat elements. Meta-gene analysis further showed that methylation decreases in tumor-prone triparous mouse were found across the entirety of the gene (Fig. 4). This decrease appeared to be specific to the tumorprone triparous mice and could indicate that the mechanisms that drive the large-scale genomic hypomethylation observed in breast tumors may be active in this early stage.
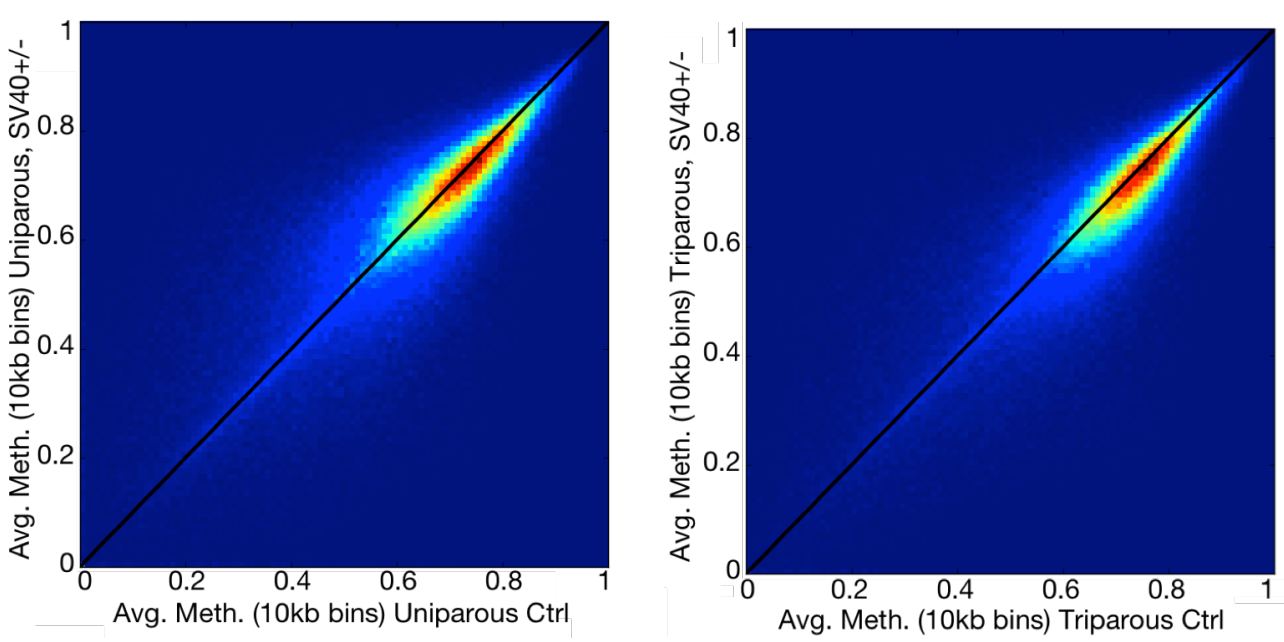

Figure 3. Correlation plot of average methylation levels computed using $10 \mathrm{~kb}$ windows for uniparous (left panel) and triparous (right panel) SV40+/- and control (Ctrl) mice.
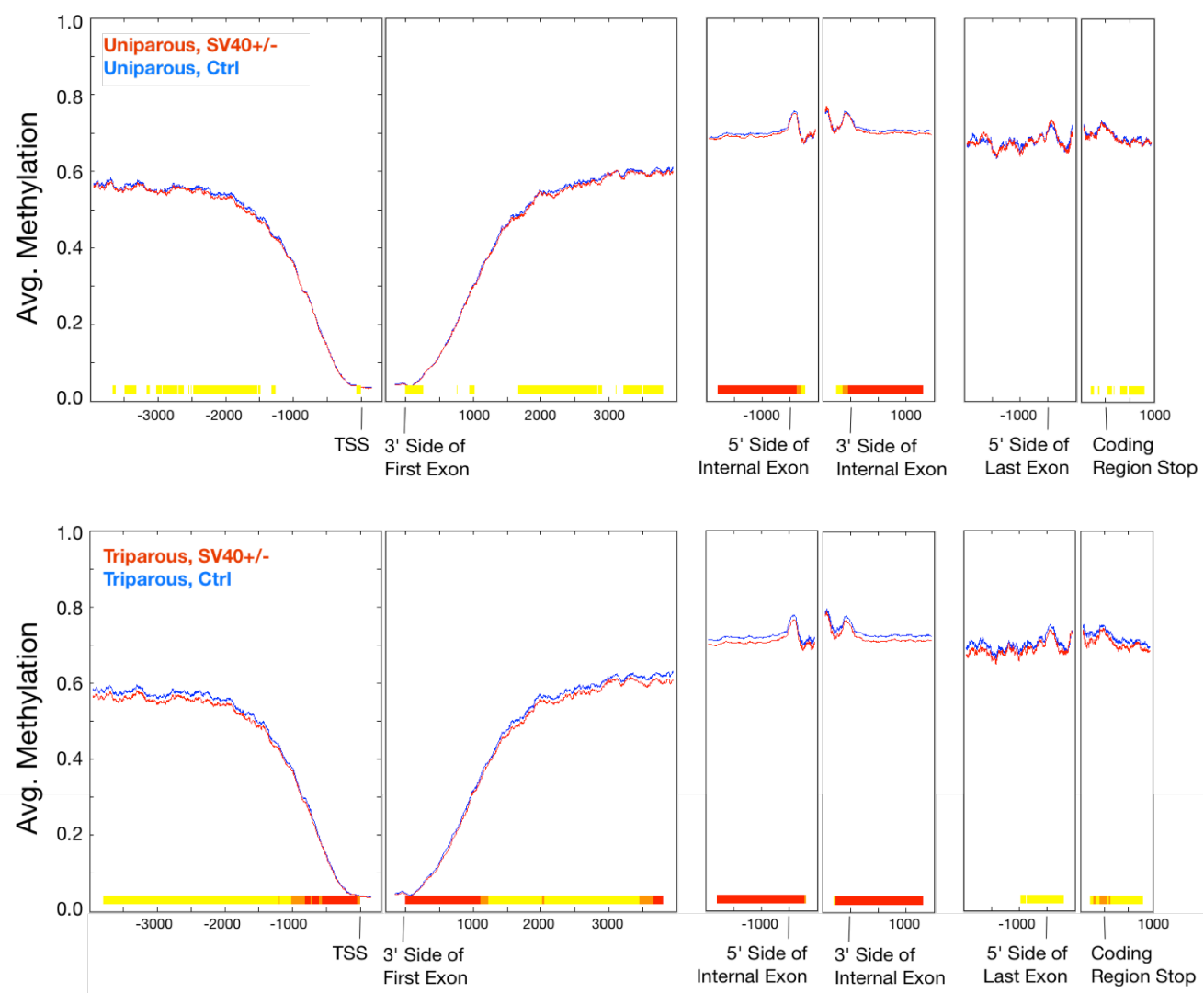

Figure 4. Meta-gene analysis of Methyl-MAPS data. The average methylation is plotted for well-annotated RefSeq genes. Red and yellow ticks indicate regions whose methylation is statistically different (red is FDR $<10^{-4}$, yellow is FDR $<0.05$ ). 
First we performed a meta-analysis to determine whether typical relationships between methylation and expression in individual samples were found in this dataset. As can be seen in Fig. 5. There is a clear inverse correlation between methylation in the region from $500 \mathrm{bp}$ to $1 \mathrm{~kb}$ to the 3 ' side of the TSS and expression quartile. This phenomenon is in line with previous reports in tumor and normal cells (Irizarry et al. 2009, VanderKraats et al. 2013). We also find a positive correlation between gene body methylation and expression quartile as has also been commonly observed (Yang et al. 2014).

Next, we used the WIMSi and DTW-kNN methods described above to analyze relationships between DNA methylation and expression at specific gene promoters. We first ran WIMSi, the unsupervised method to search for any patterns of methylation associated with transcriptional changes at gene promoters that were unique to this dataset. WIMSi analysis failed to yield any reliable genes suggesting either that any novel patterns did not occur often enough or that such patterns did not exist. Our previous analyses suggested that the dataset must contain from 15-20 examples of a pattern to be reliably detected (VanderKraats et al. 2013). Thus this analysis would indicate there are few, if any, genes with correlated promoter methylation and expression levels in these datasets.

We next ran the DTW-kNN method by building a model from Methyl-MAPS and RNA-seq data from five breast cancer cell lines. Using this model we evaluated whether there were genes that changed in the SV40 +/- versus control in both uniparous and triparous mice. We were unable to detect any genes even when we reduced the accuracy cutoff to $85 \%$. This suggests that there is not wide-spread promoter methylation contributing to the expression changes we observe in triparous mice. This was surprising, since in our analysis of five breast tumors from published TCGA data, we found between 55 and 606 genes with correlated methylation and expression levels. Analysis of the methylation of promoters of retrotransposon/coding sequence chimeric transposons will be performed with the same pipeline once the chimeric transcript analysis is completed.

Taken as a whole, while we do identify a small amount of genomic hypomethylation in tumor-prone triparous mice, these changes do not appear to be associated with increased transcriptional activity at either retrotransposons or coding genes. It also appears that the promoter methylation changes that are common in primary tumors are not found in this early stage. We conclude that the mechanisms that
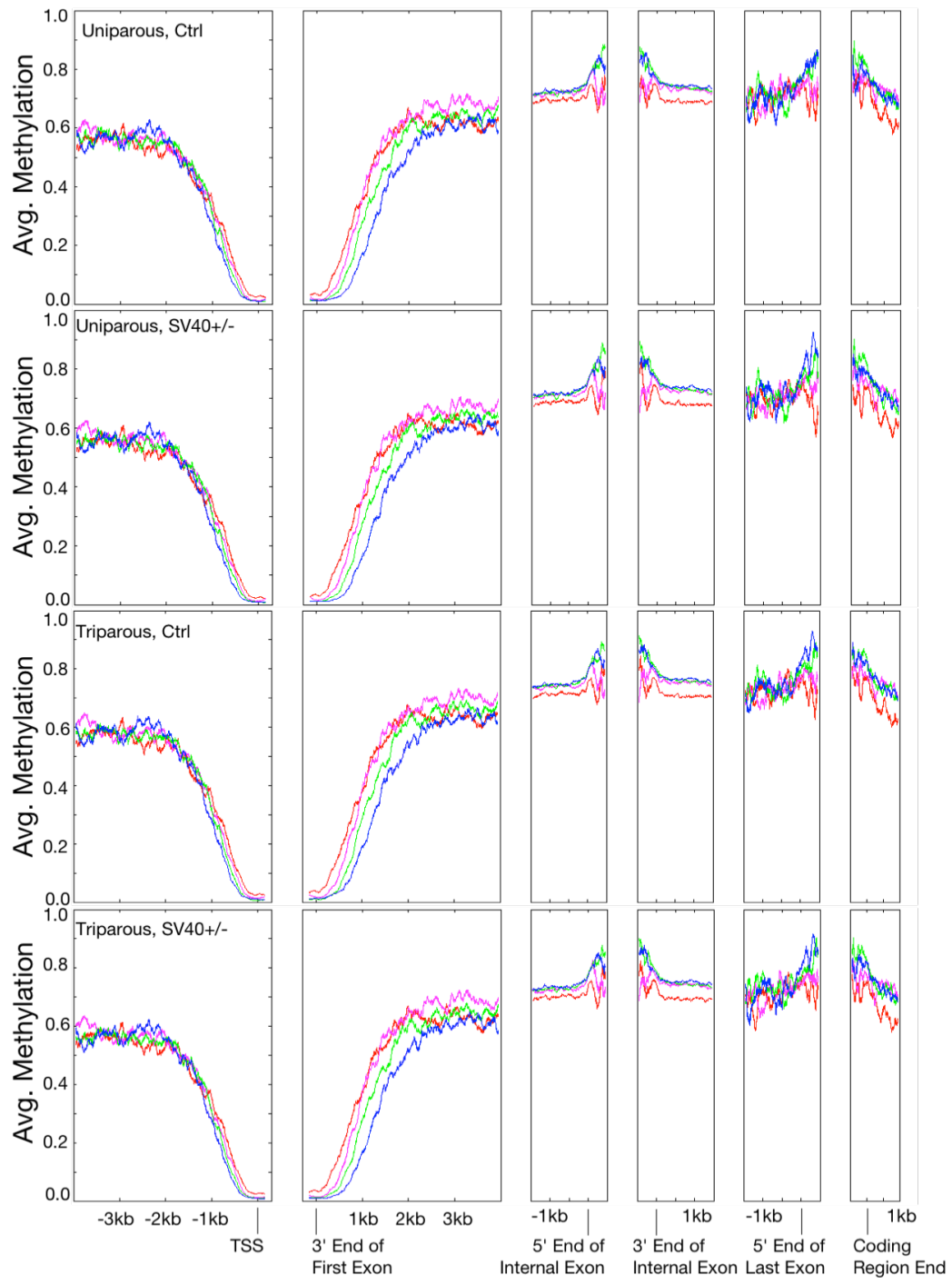

Figure 5. Meta-gene analysis by expression quartile. For each sample, average methylation was computed for genes in the lowest expression quartile (red), second lowest (magenta), second highest (green) and highest (blue). Positive correlations between methylation and expression are observed in the gene bodies (internal exons), while a negative correlation is observed 3' of the first exon. 
cause demethylation may be active and may create a permissive environment for later transcriptional changes as tumors arise. However, these methylation changes do not appear to affect transcription of retrotransposons nor coding genes at this stage. In the future, we will characterize methylation and expression from tumors arising in tumor-prone triparous mice to further understand when the large-scale remodeling of DNA methylation occurs and how these methylation changes facilitate transcriptome remodeling. 
The relevant sections from the Statement of Work are shown in the table below with corresponding goals and results. We are in the process of finalizing our analyses and performing validation of specific hypotheses this work has generated. We hope to have a manuscript prepared and submitted detailing our findings of the analysis of uniparous and tumor-prone triparous mice in the next six months.

Year 1 \& 2: Items from Statement of Work Relevant to Edwards Lab.

\begin{tabular}{|c|c|c|c|}
\hline $\begin{array}{l}\text { Year, } \\
\text { Months }\end{array}$ & Goal & & Result \\
\hline $\begin{array}{l}1 \\
1-3 \\
4-6 \\
7-9 \\
10-12 \\
\\
Y 2 \\
1-3 \\
4-6 \\
7-9 \\
10-12\end{array}$ & $\begin{array}{l}4 . \\
9 . \\
5 . \\
7 . \\
\\
8 . \\
8 . \\
9,10,11 . \\
8 .\end{array}$ & $\begin{array}{l}\text { - Set up schedule for formal monthly } \\
\text { electronic lab meeting between Peaston lab } \\
\text { and Edwards lab. And regularly hold } \\
\text { meetings. }\end{array}$ & - Completed \\
\hline $\begin{array}{l}\text { Y1 } \\
4-6\end{array}$ & 6. & $\begin{array}{l}\text { - Preliminary Methyl-MAPS analysis of pilot } \\
\text { virgin samples }\end{array}$ & $\begin{array}{l}\text { - This material was never received. Based on } \\
\text { delays in the mouse breeding and } \\
\text { conversations with the Dr. Peaston, it was } \\
\text { decided to move straight to the primary } \\
\text { analyses. }\end{array}$ \\
\hline $\begin{array}{l}\text { Y1 } \\
10-12\end{array}$ & 7. & $\begin{array}{l}\text { - Methyl-MAPS library preparation and } \\
\text { sequencing for replicate } \# 1 \text { uniparous \& } \\
\text { triparous control and tumor-prone }\end{array}$ & - Completed \\
\hline $\begin{array}{l}\text { Y2 } \\
1-3\end{array}$ & 6. & $\begin{array}{l}\text { - Preliminary Methyl-MAPS analysis of } \\
\text { replicate \#1 } \\
\text { - Review DNA loci of interest for bisulfite } \\
\text { sequencing in light of PCR results and } \\
\text { library analysis, Continue bisulfite } \\
\text { sequencing assessment of DNA loci of } \\
\text { interest }\end{array}$ & $\begin{array}{l}\text { - Completed } \\
\text { - Initial review completed. Bisulfite sequencing } \\
\text { is commencing. }\end{array}$ \\
\hline $\begin{array}{l}Y 2 \\
4-6\end{array}$ & $\begin{array}{l}3 . \\
5 .\end{array}$ & $\begin{array}{l}\text { - Methyl-MAPS library preparation and } \\
\text { sequencing for replicate } \# 2 \text { uniparous \& } \\
\text { triparous control and tumor-prone } \\
\text { - Library analyses replicate \#2 and } \\
\text { preliminary comparisons with replicate \#1 }\end{array}$ & $\begin{array}{l}\text { - Material was received. We encountered } \\
\text { problems with library construction due to the } \\
\text { low amount of DNA provided. We are } \\
\text { working to salvage these samples and plan to } \\
\text { sequence shortly. }\end{array}$ \\
\hline $\begin{array}{l}2 \\
7-9\end{array}$ & 4. & $\begin{array}{l}\text { - Methyl-MAPS library preparation and } \\
\text { sequencing for replicate } \# 3 \text { uniparous \& } \\
\text { triparous control and tumor-prone }\end{array}$ & $\begin{array}{l}\text { - Material was received. Based on results from } \\
\text { replicate } \# 2 \text {, there is insufficient material to } \\
\text { construct the libraries for sequencing. A } \\
\text { strategic decision was made to hold the } \\
\text { material for validation, rather than attempt } \\
\text { library construction. }\end{array}$ \\
\hline $\begin{array}{l}\text { Y2 } \\
10-12\end{array}$ & $\begin{array}{l}2 . \\
3 . \\
5 .\end{array}$ & $\begin{array}{l}\text { - Finalize data analysis } \\
\text { - Prepare a report for publication of the results }\end{array}$ & $\begin{array}{l}\text { Analysis pipelines have been developed and } \\
\text { are in place. Initial analysis is complete. } \\
\text { Deeper analysis will be finished shortly. } \\
\text { Reports will be finalized once the analyses } \\
\text { and validation are finished. }\end{array}$ \\
\hline
\end{tabular}




\section{Key Research Accomplishments}

- We developed new computational tools to combine genome-wide expression and methylation data to output a list of genes where methylation likely contributes to their silencing or activation.

- We performed genome-wide methylation and expression profiling of SV40+/- and control uni- and triparous mice.

- We found a small amount of genomic demethylation specific to tumor-prone triparous mice that was not accompanied with corresponding changes in the transcriptome. These changes indicate that the mechanisms driving demethylation in breast tumors may have activated, even though they may not yet contribute to transcriptome remodeling at this early stage. 


\section{Reportable Outcomes}

\section{Manuscripts}

Vanderkraats ND, Hiken JF, Decker KF, Edwards JR. (2013) Discovering high-resolution patterns of differential DNA methylation that correlate with gene expression changes. Nucleic Acids Res. 41:6816-6827.

Lund K, Cole JJ, VanderKraats ND, McBryan T, Pchelintsev NA, Clark W, Copland M, Edwards JR, Adams PD. (2014) DNMT inhibitors reverse a specific signature of aberrant promoter DNA methylation and associated gene silencing in AML. Genome Biol. 15:406.

Cruickshanks HA, McBryan T, Shah PP, Nelson DM, Donahue G, VanderKraats ND, Edwards JR, Berger SL, Adams PD. (2013) Features of the cancer epigenome are acquired as primary human cells approach senescence. Nature Cell Biology. 15:1495-1506.

Boulard M, Edwards JR, Bestor TH. (2015) "FBXL10 protects Polycomb-bound genes from Hypermethylation.” Nat. Genetics. In press.

\section{Invited Manuscripts}

Bestor TH, Edwards JR, Boulard M. (2014) Notes on the role of dynamic DNA methylation in mammalian development. PNAS. Published ahead of print November 3, 2014, doi:10.1073/pnas.1415301111.

\section{Abstracts}

Schlosberg CE, VanderKraats ND, Hiken JF, Weinberger KQ, Ju T, Edwards JR. (2014) "Modeling complex patterns of differential DNA methylation that strongly associate with gene expression changes." 22nd Annual International Conference on Intelligent Systems for Molecular Biology, July 13-15. 


\section{Conclusion}

The major conclusion from this work is that at this early stage of tumor development, we find only a small amount of genome-wide hypomethylation in tumor-prone triparous mice and do not observe widespread DNA methylation changes that are commonly found in tumors. Correlated methylation changes at promoters and expression changes are found at hundreds of genes in primary human tumors, but none were observed in the tumor-prone mice in this study. Likely the mechanisms that lead to the genome-wide hypomethylation observed in primary tumors have activated, but they are not impacting transcription of retrotransposons nor coding genes at this early stage.

Expression analyses indicate that the tumor-prone triparous mice experience altered transcription of several hundred transcripts. Many of these transcripts are found in cell cycle control and could represent the early inactivation of checkpoints to control proliferation. There does not appear to be wide-spread methylation or expression changes in retrotransposon sequences, indicating that these likely play little to no role in this stage.

During the course of this proposal we have developed a new set of computation tools that potentially have broad significance beyond the work here. The computational tools we have developed are designed to work with annotated genes as we have outlined, but can also be expanded to any transcriptional unit with a known TSS and known expression value. Further we have demonstrated how they can be directly used to study which genes are correlated, and thus potentially regulated, by DNA methylation in cancer cells. We have also shown how we can use these tools to identify genes that are potentially up-regulated in direct response to demethylating agents such as 5-azacitidine and Decitabine. While DNA demethylating agents have primarily been employed to treat Myelodysplastic syndrome and AML, recently these drugs have been explored as potential therapeutics in solidtumors such as lung and breast cancer. We believe this work has the potential to shed light on which patients have genes that can be potentially re-activated by these drugs, and thus which patients can potentially benefit from this line of therapy. 


\section{References}

Berman B, et al. (2012) Regions of focal DNA hypermethylation and long-range hypomethylation in colorectal cancer coincide with nuclear lamina-associated domains. Nature Genetics 44, 40-46.

Cruickshanks HA, McBryan T, Shah PP, Nelson DM, Donahue G, VanderKraats ND, Edwards JR, Berger SL, Adams PD. (2013) Features of the cancer epigenome are acquired as primary human cells approach senescence. Nature Cell Biology. 15, 1495-1506.

Hansen KD, Langmead B \& Irizarry RA. (2012) BSmooth: from whole genome bisulfite sequencing reads to differentially methylated regions. Genome biology 13, R83.

Hon GC, et al. (2012) Global DNA hypomethylation coupled to repressive chromatin domain formation and gene silencing in breast cancer. Genome Research. 22, 246-258.

Huang DW, et al. (2009) Systematic and integrative analysis of large gene lists using DAVID Bioinformatics Resources. Nat Protoc. 4, 44 -57.

Irizarry RA, et al. (2009) Genome-wide methylation analysis of human colon cancer reveals similar hypo- and hypermethylation at conserved tissue-specific CpG island shores. Nat. Genetics. 41, 178-186.

Lou S, et al. (2014) Whole-genome bisulfite sequencing of multiple individuals reveals complementary roles of promoter and gene body methylation in transcriptional regulation. Genome Biology 15, 408.

Lund K, Cole JJ, VanderKraats ND, McBryan T, Pchelintsev NA, Clark W, Copland M, Edwards JR, Adams PD. (2014) DNMT inhibitors reverse a specific signature of aberrant promoter DNA methylation and associated gene silencing in AML. Genome Biol. 15, 406.

Robinson MD, McCarthy DJ, Smyth GK (2010). “edgeR: a Bioconductor package for differential expression analysis of digital gene expression data.” Bioinformatics, 26, pp. -1.

Trapnell C, Pachter L, Salzberg SL. (2009) TopHat: discovering splice junctions with RNA-Seq. Bioinformatics 25, 1105-1111.

Vanderkraats ND, Hiken JF, Decker KF, Edwards JR. (2013) Discovering high-resolution patterns of differential DNA methylation that correlate with gene expression changes. Nucleic Acids Res 41, 68166827.

Yang X, et al. (2014) Gene body methylation can alter gene expression and is a therapeutic target in cancer. Cancer Cell. 26, 577-590. 


\section{Appendix}

Vanderkraats ND, Hiken JF, Decker KF, Edwards JR. (2013) Discovering high-resolution patterns of differential DNA methylation that correlate with gene expression changes. Nucleic Acids Res. 41:6816-6827. 


\title{
Discovering high-resolution patterns of differential DNA methylation that correlate with gene expression changes
}

\author{
Nathan D. VanderKraats, Jeffrey F. Hiken, Keith F. Decker and John R. Edwards* \\ Center for Pharmacogenomics, Department of Medicine, Washington University School of Medicine, $660 \mathrm{~S}$. \\ Euclid Ave, Campus Box 8220, St. Louis, MO 63110, USA
}

\begin{abstract}
Methylation of the CpG-rich region (CpG island) overlapping a gene's promoter is a generally accepted mechanism for silencing expression. While recent technological advances have enabled measurement of DNA methylation and expression changes genome-wide, only modest correlations between differential methylation at gene promoters and expression have been found. We hypothesize that stronger associations are not observed because existing analysis methods oversimplify their representation of the data and do not capture the diversity of existing methylation patterns. Recently, other patterns such as $\mathrm{CpG}$ island shore methylation and long partially hypomethylated domains have also been linked with gene silencing. Here, we detail a new approach for discovering differential methylation patterns associated with expression change using genome-wide high-resolution methylation data: we represent differential methylation as an interpolated curve, or signature, and then identify groups of genes with similarly shaped signatures and corresponding expression changes. Our technique uncovers a diverse set of patterns that are conserved across embryonic stem cell and cancer data sets. Overall, we find strong associations between these methylation patterns and expression. We further show that an extension of our method also outperforms other approaches by generating a longer list of genes with higher quality associations between differential methylation and expression.
\end{abstract}

\section{INTRODUCTION}

DNA methylation is an important factor in transcriptional regulation, playing a role in genomic imprinting, $\mathrm{X}$-inactivation, retrotransposon silencing and the control of tissue-specific genes during differentiation (1). DNA methylation patterns are frequently altered in tumors (2), and there is great interest in understanding how changes to these patterns contribute to human disease (3). Even so, how alterations to DNA methylation affect gene transcription remains poorly characterized. Over $60 \%$ of genes have a $\mathrm{CpG}$-rich region, termed a $\mathrm{CpG}$ island, overlapping their promoter (4). Classically, it is thought that hypermethylation of promoter-associated $\mathrm{CpG}$ islands silences transcription. However, it was recently shown that cancer- and tissue-specific methylation variation in adjacent regions, termed $\mathrm{CpG}$ island shores, is also associated with gene expression change (5). Additionally, genes are more likely to be repressed when they are located in partially methylated domains (6) or long hypomethylated domains $(7,8)$ in cancer.

Techniques such as whole-genome bisulfite sequencing (WGBS) (9) and Methyl-MAPS (10) have recently been developed to map methylation at single-base resolution genome-wide. Methods to interpret this data, however, are lacking. Current computational techniques are mostly concerned with the visualization of genome-level correlations between DNA methylation and other epigenetic marks, or with the identification of regions that are differentially marked between samples [recently reviewed in (11)]. These tools have elucidated the genomic organization of these marks, but they do not sufficiently address how changes at individual loci associate with and potentially affect function.

The most common approach for characterizing methylation changes between two samples uses a sliding window to identify differentially methylated regions (DMRs) $(7,9)$. A gene with a hypermethylated DMR near its promoter is assumed to exhibit a decrease in expression, while a gene near a hypomethylated DMR should exhibit an increase in expression. In practice, the Pearson correlation coefficient between the methylation level of the DMR and the expression of its associated gene is around $-0.3(11,12)$. It has been assumed that better anticorrelation is precluded owing to noise from experimental error, mixed cellular

*To whom correspondence should be addressed. Tel: +1 314362 6935; Fax: +1 314362 8844; Email: jedwards@dom.wustl.edu

(C) The Author(s) 2013. Published by Oxford University Press.

This is an Open Access article distributed under the terms of the Creative Commons Attribution Non-Commercial License (http://creativecommons.org/licenses/ by-nc/3.0/), which permits non-commercial re-use, distribution, and reproduction in any medium, provided the original work is properly cited. For commercial re-use, please contact journals.permissions@oup.com 
populations, copy number variations, chromatin modifiers or other regulation events. Another explanation, however, is that contemporary analysis methods are not sophisticated enough to recognize relationships involving more complex methylation patterns. Existing approaches summarize their representation of methylation change in the promoter region to simplify analysis, but this sacrifices potentially important spatial information contained in the locations of the constituent sites.

To discover DNA methylation changes that associate with gene expression changes, we propose a new method that uses the entire differential methylation profile in the vicinity of a gene's promoter (Figure 1). We represent the differential methylation for a fixed area around each gene's transcription start site (TSS) as a continuous curve, or signature, capturing the shape of the methylation changes. We then apply a curve similarity metric, the discrete Fréchet distance, to compare differential methylation signatures for all genes. Using an unsupervised clustering technique, we arrange the signatures according to their shapes and identify which clusters of genes exhibit statistically significant changes in expression. Generalized patterns of differential methylation can be extrapolated from the resulting clusters. Because the approach is unsupervised, no assumptions need to be made about the direction of a correlation between methylation and expression. Although designed for pattern discovery, the method is easily extended to identify a list of genes potentially regulated by methylation. These gene lists are of markedly greater length and have higher quality associations between differential methylation and expression than those generated by existing methods. A current implementation of the approach can be found on the project website at http://epigenomics.wustl.edu/WIMSi/.

\section{MATERIALS AND METHODS}

\section{Methylation signatures}

Using WGBS or Methyl-MAPS data (single-base resolution) to compare methylation patterns between different genes' promoter regions is complicated by the variability in the locations of their CpGs. To enable comparisons between genes, we standardize the representation of differential methylation data for each gene by creating a methylation signature across a fixed-width region centered at the TSS (Figure 1). In a single sample, the methylation level at each probed $\mathrm{CpG}$ is represented as a continuous value between 0 and 1 , denoting fully unmethylated and methylated, respectively. To compare methylation between two samples, we subtract these values at each site to produce a differential methylation score that ranges from -1 to 1 , denoting complete hypomethylation and hypermethylation, respectively.

We create a gene's methylation signature on a fixedwidth target region by interpolating the differential methylation scores using piecewise cubic Hermite interpolation (Figure 1B and C) across all $\mathrm{CpG}$ sites within the region and between one and five $\mathrm{CpG}$ sites flanking the region on either side. Because methylation is highly correlated over short distances (Supplementary Figure S1) (13), interpolation provides a suitable estimate for differential methylation in areas with missing data. Such missing data points can occur owing to insufficient coverage or experimental limitations (e.g. data collected only at specific restriction sites). Interpolated values always lie between -1 and 1. Regions with fewer than five $\mathrm{CpG}$ sites with sufficient coverage and regions with fewer than one flanking site per side are discarded. We also discard regions containing no $\mathrm{CpG}$ sites with absolute differential methylation $>0.2$. Lastly, we apply Gaussian smoothing on the curves $(\sigma=50 \mathrm{bp})$ to help moderate noise due to experimental artifacts such as missing or inaccurate measurements. One advantage of using a combination of interpolation and smoothing is that it improves performance of the method for low coverage data. Previously it was shown that statistical smoothing was an effective method to analyze low coverage WGBS data (14). The resulting methylation signatures for each gene are bounded between -1 and 1 on a fixed region relative to the TSS.

\section{Determining clusters of methylation signatures with significant expression changes}

We compare methylation signatures between genes using the discrete Fréchet distance, also known as the coupling distance $(15,16)$. The Fréchet distance is informally known as the dog-man distance because it represents the minimum length of leash necessary for a person traversing one curve to walk a dog along another, assuming neither party is allowed to walk backwards. The Fréchet metric is advantageous because it is efficiently computable, while still taking into account the entire course of the curves. In particular, it appeals to an intuitive notion of similarity between methylation signatures in that two curves with similar shape will have a low distance, even if one curve is shifted slightly from the other relative to the TSS (Supplementary Figure S2). Because Fréchet distance is calculated in Euclidean space, a scaling parameter must specify the relationship between the x-axes of the curves, in bp, and the y-axes, in differential methylation level. Intuitively, this parameter controls how far peaks in the $\mathrm{y}$-direction are allowed to slide across the $\mathrm{x}$-axis and still be identified as similar between two genes.

Formally, a methylation signature, or curve, can be described as a continuous mapping $\mathrm{f}:[0,1] \rightarrow\left[0, \mathrm{n}_{\mathrm{b}}\right] \times[-1,1]$ where $n_{b}$ is the fixed length of the region in base pairs. For two curves $\mathrm{A}$ and $\mathrm{B}$, the Fréchet distance is defined as follows:

$$
\delta_{\text {Frechet }}(A, B)=\inf _{\alpha, \beta} \max _{t} d(A(\alpha(t)), B(\beta(t)))
$$

where $t \in[0,1], d(x, y)$ is the Euclidean distance between $x$ and $y$, and $\alpha(t)$ and $\beta(t)$ are continuous, monotonically increasing functions from $[0,1]$ to $[0,1]$ such that $\alpha(0)=\beta(0)=0$ and $\alpha(1)=\beta(1)=1$. For any two differential methylation signatures, we calculate similarity using the coupling distance, which can be computed on polygonal curves in quadratic time using a dynamic programming algorithm (16). Each continuous interpolated curve is converted into a polygonal curve 
A

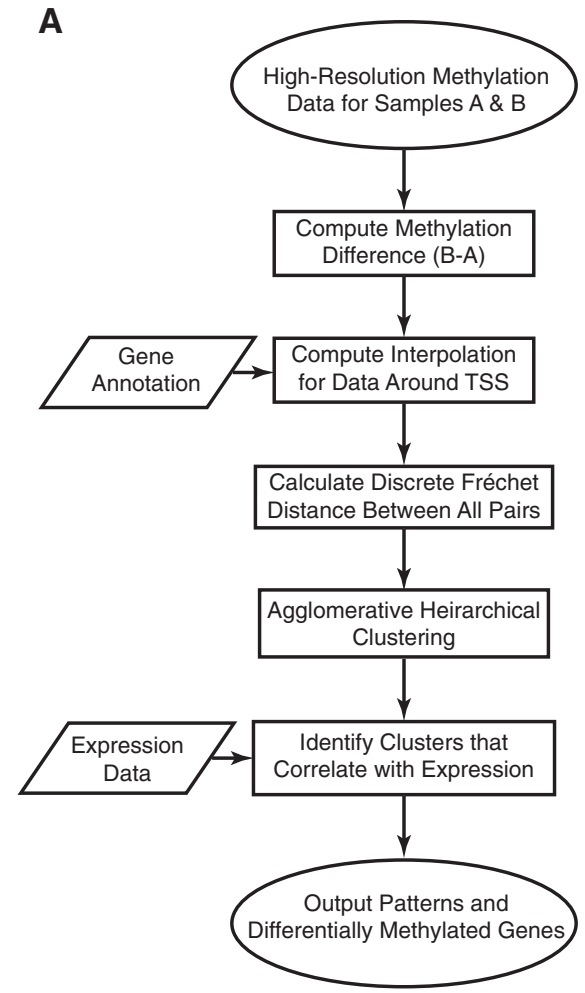

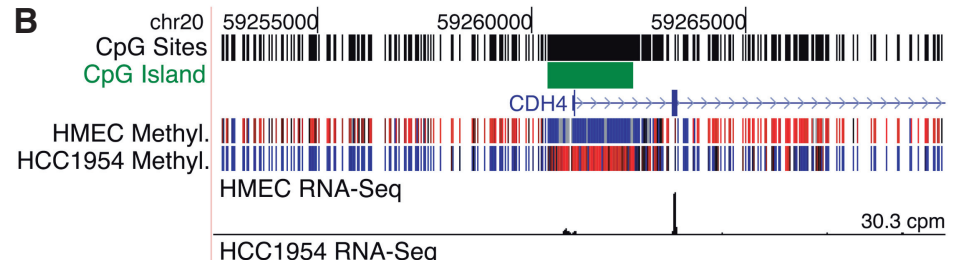
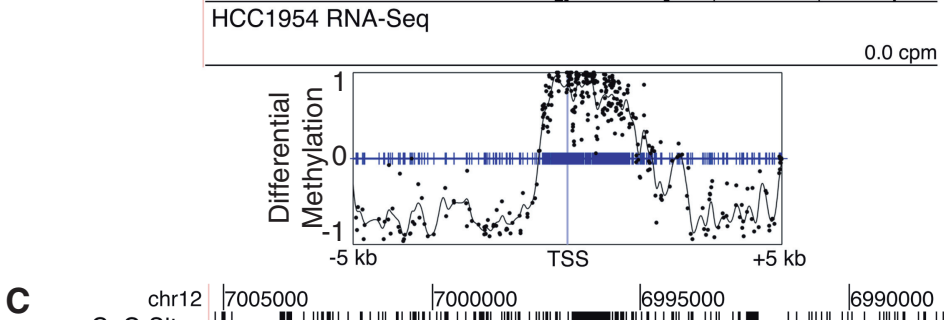
Cpg Sites |||| || | |||||| | |||| || |||||||||||||| |||||||||||||| |||||||||||||| || || ||| || || ||| || || | |||||| || || CpG Island

HMEC Methyl.

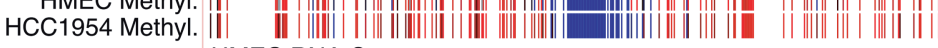
HMEC RNA-Seq

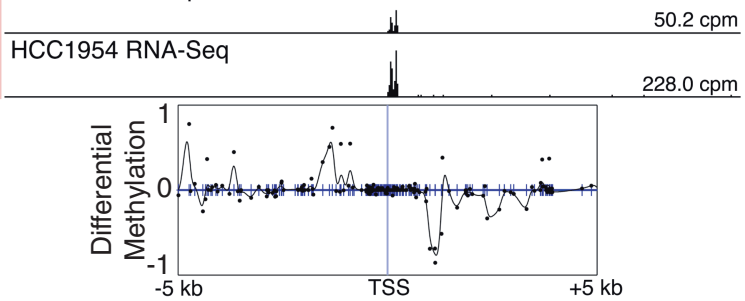

Figure 1. Method overview and example methylation signatures. (A) Overview of the approach for associating spatially similar DNA methylation changes with corresponding changes in transcription. (B, C) Example methylation signatures from HMEC-HCC1954 WGBS data for the tumor suppressor gene CDH4 and the LPCAT3 gene. Conversion of methylation data to signatures allows direct comparison of the data between genes with different distributions of $\mathrm{CpG}$ sites relative to their TSSs. Top panels show methylation (blue is unmethylated and red is methylated) and RNASeq expression data on the UCSC genome browser. Bottom panels show interpolated and smoothed methylation signatures (black curve) that are used to calculate the discrete Fréchet distance. Blue tick marks show locations of all $\mathrm{CpG}$ sites. Black dots mark experimentally measured differences in methylation between the two samples.

by averaging every $10 \mathrm{bp}$, yielding $\operatorname{ceil}\left(n_{b} / 10\right)$ vertices for the $n_{b}$ bases in the target region. For sparsely sampled areas, this fixed resolution greatly limits the discretization error between our discrete Fréchet distance and the continuous version because this error is bounded by the maximum edge length for each pair of curves (17). Averaging every $10 \mathrm{bp}$ allows the algorithm to run faster than sampling every $2 \mathrm{bp}$. Because the averaging interval is considerably smaller than the width of the Gaussian smoothing kernel, it has little effect on the resulting distances. We compared the results of clustering for several experiments using $10 \mathrm{bp}$ averaging and dinucleotide sampling, and found no differences in the type and number of patterns found for the HMEC-HCC1954 data set. By default, scaling between the $\mathrm{x}$ - and $\mathrm{y}$-axes was set such that $2500 \mathrm{bp}$ along the $\mathrm{x}$-direction was equivalent to one unit of differential methylation in the $y$-direction. We tested a large number of values for this ratio. Manual inspection of resulting clusterings showed that the final set of patterns discovered was not substantially altered despite moderate changes to the scaling ratio.

Methylation signatures are arranged using unsupervised complete-linkage agglomerative hierarchical clustering based solely on the discrete Fréchet distance between curve pairs. After clustering, we introduce the differential expression data to identify clusters of genes with similar expression differences. Expression data can be obtained through any method, including RNA-Seq and expression array analysis. To focus on genes for which differential methylation and expression could be related, we consider only genes with greater than 2-fold expression change.

We identify clusters from the dendrogram where methylation is significantly associated with expression as follows. For each cluster, we evaluate the likelihood that the observed group of expression values is atypical compared with the distribution of expression values over the entire training set [a dendrogram on $n_{s}$ signatures contains exactly $\left(n_{s}-1\right)$ clusters]. We determine significance using a two-sample Kolmogorov-Smirnov test between the set of differential expression values in each cluster and the entire population of expression values. The false discovery rate is controlled at 0.05 using the BenjaminiHochberg procedure. This estimate of significance is conservative because one sample is a subset of the other.

After all statistically significant clusters are identified, we select a set of nonoverlapping clusters using an iterative algorithm to define the trade-off between grouping similar patterns together versus breaking them into distinct clusters. We seek a balance between the selection of 
larger clusters that embody more general patterns and the conformity between differential expression values, called purity. The purity of a set of genes is defined as the fraction of genes that have expression change in the same direction as the majority. The full process is described in the Supplementary Methods. We used a minimum purity of 0.85 unless otherwise stated.

\section{Generating a gene list}

To produce a list of genes with associated differential methylation and expression, we ran the discovery method on a set of overlapping $5 \mathrm{~kb}$ regions centered at a fixed set of locations around the TSS (Figure 6A). The scaling factor between the $\mathrm{x}$ - and $\mathrm{y}$-dimensions, minimum cluster purity and all interpolation parameters were the same as previously described. We used 22 regions, spanning an area from $[-25 \mathrm{~kb}, 25 \mathrm{~kb}]$ relative to the TSS. The area [ $-10 \mathrm{~kb}, 10 \mathrm{~kb}]$ relative to the TSS was covered more densely because this was where the majority of relevant differential methylation features were observed by the pattern discovery tool. The leftmost boundaries (in $\mathrm{kb}$, relative to the TSS) were: $-25,-20,-15,-10,-9,-8$, $-7,-6,-5,-4,-3,-2,-1,0,1,2,3,4,5,10,15$ and 20. For each region, we recorded the set of genes identified as positives (i.e. changing in the same direction as the majority of their cluster). Genes that are identified for at least $m$ regions are added to the final list. Unless otherwise stated, an $m$ of two regions was used.

\section{RESULTS}

\section{Pattern discovery in high-resolution methylation data}

We evaluated our technique using three primary and nine additional comparisons (17 total data sets) with high-resolution methylation and RNA-Seq expression data (Supplementary Table S1). The first primary data set was WGBS of nontumorigenic human mammary epithelial cells (HMEC) and breast cancer cells (HCC1954) (6) containing methylation data for $84.7 \%$ of genomic CpGs with a coverage level of at least 10 in each sample (Supplementary Table S1, GEO: GSE29127). We focused many of the analyses in this article on this HMECHCC1954 data set because it has high coverage and contains examples of all the methylation patterns we discovered (Supplementary Data 1). To examine how the method performed on a lower coverage data set, we examined WGBS data for H1 embryonic stem (ES) cells and IMR90 fetal lung fibroblasts (9) (GEO: GSE16256). While the genomic coverage level of this data was high, the data was sparsely sampled at promoters: $<40 \%$ of CpGs had coverage of at least 10 (Supplementary Figure $\mathrm{S} 3)$. By including all $\mathrm{CpGs}$ with coverage as low as a single read, the data covered $93.5 \%$ of genomic CpGs. However, methylation scores are expected to be less accurate in regions with lower sampling. WGBS data was processed and methylation scores computed as in (6). Analysis was limited to only $\mathrm{CpG}$ methylation (complete results are in Supplementary Data 2). Lastly, to validate our findings using data from an alternative experimental method, we generated Methyl-MAPS data from MCF7 and T47D breast cancer cells. Methyl-MAPS uses methylation-sensitive and -dependent restriction enzyme digests followed by high-throughput sequencing to identify methylation levels at individual CpGs (10). Libraries were constructed, sequenced and analyzed as in (10) (see Supplementary Methods for further details). We limited our analysis to sites interrogated by both digests, which included $24.9 \%$ of genomic CpGs with coverage of at least five to ensure an adequate number of $\mathrm{CpGs}$ with data around each promoter. Expression data for each sample came from poly(A) selected RNA-Seq experiments (see Supplementary Methods for further details). All MethylMAPS and RNA-Seq data are available from GEO, accession GSE45337. Complete results are in Supplementary Data 3.

In addition to these three primary comparisons, we applied our method to WGBS and RNA-Seq data comparing H9 ES cells $(18,19)$ to IMR90 cells, H1 cells to four H1-derived differentiated cell types, female adipose-derived stem cells (ADS) to ADS-derived adipocytes and ADS-derived induced pluripotent stem cells (ADS-iPSCs) (19) and primary mouse ES cells to isolated sperm and oocytes (20).

We selected an initial region for the discovery of differential methylation patterns that associate with expression changes based on two criteria. First, average $\mathrm{CpG}$ density increases roughly $2 \mathrm{~kb}$ upstream and downstream of the TSS, suggesting that sites in this region may have regulatory importance (21). Second, increased variability of differential methylation in $\mathrm{CpG}$ island shores, defined as the regions up to $2 \mathrm{~kb}$ away from a $\mathrm{CpG}$ island, has been linked to differential expression (5). To search for patterns across this entire area, we chose a conservative initial region of $10 \mathrm{~kb}$ centered on the TSS. Using a cluster purity threshold of 0.85 , we identified 27 clusters that were significantly correlated with differential expression, containing 519 genes, in the HMEC-HCC1954 data set (Figure 2; complete clustering results are in Supplementary Data 1). A cartoon depiction of each of the patterns observed is shown in Figure 3. Applying our method to the H1-IMR90 and MCF7-T47D MethylMAPS data sets showed that our method could still identify clusters corresponding to each of the patterns discovered in the higher quality HMEC-HCC1954 data set even with low promoter coverage or substantially reduced sampling (Supplementary Data 2 and 3). It is likely that interpolation and Gaussian smoothing are helpful for analyzing low coverage data. Limiting HMEC-HCC1954 data to only sites probed by Methyl-MAPS showed the data reduction had no impact on the ability to detect each of the identified patterns. As a negative control, we randomly scrambled the expression values for all genes in each data set; any cluster identified as significant was a false positive. For 1000 random permutations, our technique identified a false-positive cluster in $1.7-2.3 \%$ of the experiments (Supplementary Table S2).

\section{Patterns overlapping the TSS}

From the resulting sets of significant clusters, we sought to characterize the common features of the methylation 
A
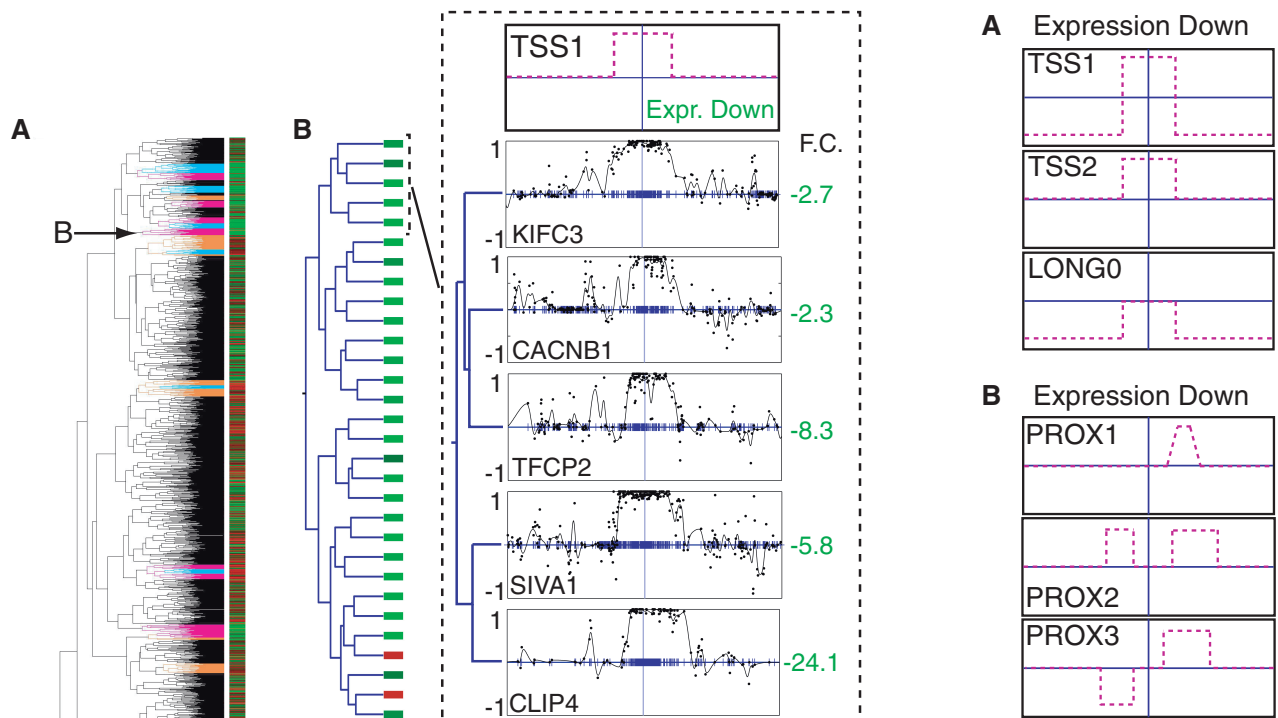

B

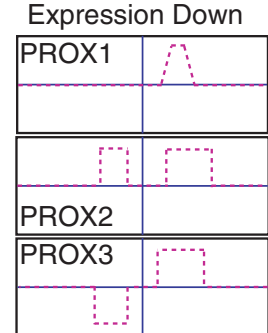

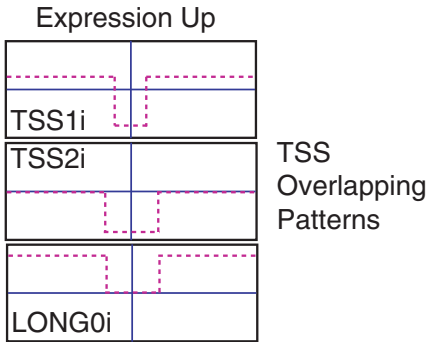

Expression Up

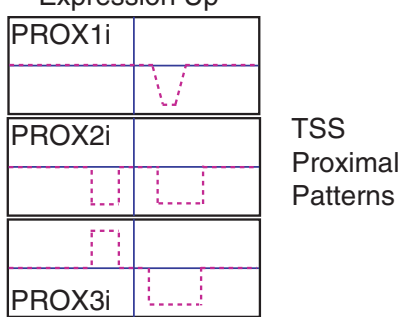

Figure 3. Summary of the diverse patterns found in the data sets analyzed. One version of each pattern is shown to the left with its corresponding inverted pattern to the right. (A) Patterns that overlap the TSS (TSS1, TSS2 and LONG0), and their respective inverted versions (TSS1i, TSS2i, and LONG0i). (B) Patterns proximal to the TSS all include a region of change downstream of the TSS and are separated by their upstream differences. Shown are PROX1, PROX2, PROX3 and their respective inverted versions: PROX1i, PROX2i and PROX3i.

signatures that may be responsible for the observed relationships with expression change. As expected, many clusters contained patterns with a region of strong hyperor hypomethylation spanning the TSS that negatively correlated with expression change (Figure 2B). Further inspection of HMEC-HCC1954 data revealed several distinct patterns overlapping the TSS. After rerunning our method using methylation signatures based on a $30 \mathrm{~kb}$ region centered at the TSS, three distinct patterns emerged (Figure 3A): a hypermethylated region at the TSS surrounded by long hypomethylated domains (TSS1; Figure 4A), a hypermethylated region at the TSS set in a region with invariant methylation levels (TSS2; Figure 2B) and a pattern of long hypomethylated domains, but with no change in methylation at the TSS (LONG0; Figure 4F). The rarest pattern, LONG0, was also observed in the H9IMR90 (Supplementary Figure S7) and H1-IMR90 (Supplementary Data 2) comparisons. Hypomethylated domains associated with these patterns (TSS1, LONG0) extend up to several $\mathrm{Mb}$ in both directions (Figure 4C). In HMEC-HCC1954 data, we found a hypomethylated region at the TSS set in a hypermethylated region (TSS1i; Figure 4B). While not all patterns were observed in every comparison we analyzed, the overall set of patterns was common across all data sets. For instance, MCF7-T47D and H1-IMR90 data sets show an inverted TSS2 pattern (TSS2i; Supplementary Data 2 and 3). IMR90 fibroblasts exhibit a TSS1 pattern relative to $\mathrm{H} 1$ stem cells (Supplementary Data 2), although the hypomethylated domains in fibroblasts are much smaller (Figure 4E). It has been suggested that the observed positive correlation 

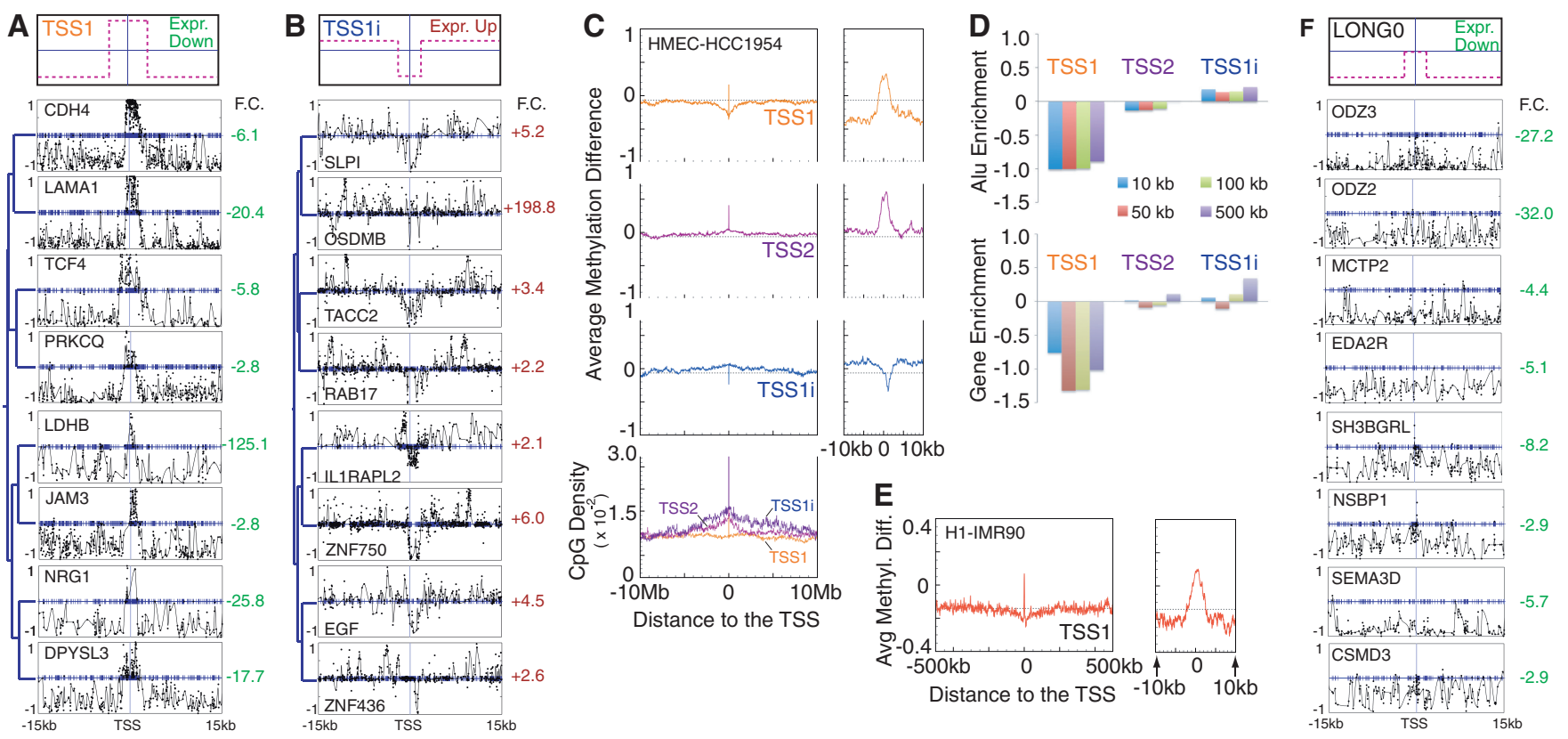

Figure 4. Analysis of patterns overlapping the TSS. (A, B) Top panel is a cartoon depicting the relevant pattern. Bottom panel shows an example subcluster from an HMEC-HCC1954 cluster. Clustering was performed on $30 \mathrm{~kb}$ regions. F.C. denotes expression fold change; green indicates downregulation, red indicates upregulation. (A) Subcluster characterized by hypermethylation at the TSS set in a long hypomethylated domain (TSS1). The entire cluster is in Supplementary Figure S6. (B) Subcluster characterized by hypomethylation at the TSS set in a long hypermethylated domain (TSS1i). The entire cluster is in Supplementary Figure S8. (C) Average differential methylation and CpG density for genes from clusters identified as exhibiting three TSS patterns (TSS1, TSS1i and TSS2). Example signatures for each of the three patterns are shown in parts (A), (B) and Figure 2B. (D) Alu elements and RefSeq genes are depleted in the regions around genes with the TSS1 pattern. No enrichment or depletion of other repeats was found (Supplementary Figure S9). (E) The TSS1 pattern is also found in the H1-IMR90 comparison (Supplementary Data 2). (F) Example subcluster showing the LONG0 pattern from the HMEC-HCC1954 comparison. The entire cluster is in Supplementary Figure S6.

between gene-body methylation and expression may be due to similar domains (8). Inspection of individual genes with the LONG0 pattern revealed that promoters were hypomethylated in both samples, leading us to speculate that long hypomethylated domains could contribute to gene silencing, possibly through the recruitment of factors that lead to the formation of repressive chromatin (6). Clusters representing the inverse patterns - a hypomethylated region at the TSS set in either long hypermethylated or invariant regions -were also observed (TSS1i, TSS2i; Figure 4B).

\section{Patterns proximal to the TSS}

In addition to patterns with differential methylation across the TSS, we identified multiple clusters in all data sets characterized by a change in methylation downstream of the TSS (Figure 2C, Figure 5A and B). Analysis of all genes in clusters associated with this pattern indicated that it predominantly occurs within $3 \mathrm{~kb}$ of the TSS (Figure 5C). This $3^{\prime}$ pattern was observed in several distinct clusters due to variations in other parts of the differential methylation curves (see examples in Figure 5A and B). The TSSproximal patterns found by our discovery method are in agreement with the variation in methylation found at $\mathrm{CpG}$ island shores. Interestingly though, we find no significant association between the proximal methylation patterns and whether promoters are classified as $\mathrm{CpG}$-rich or -poor (Supplementary Figure S10). This may suggest that these proximal methylation changes are not confined to island shores, or it may be due to the fact that the patterns we discover are anchored to the TSS.

Although some clusters with $3^{\prime}$ patterns also displayed methylation change upstream of the TSS, no independent relationship was observed between a $5^{\prime}$ pattern and differential expression (Figure 5A and B). Differential methylation downstream of the TSS was consistently linked with expression change, regardless of upstream hyper- or hypomethylation. To further probe whether distinct correlative patterns occur upstream of the TSS, we ran our method using signatures defined on the region from $-5 \mathrm{~kb}$ to the TSS. The majority of identified clusters were characterized by changes in methylation at the TSS. Genes in clusters with methylation changes $5^{\prime}$ of the TSS often had correlative $3^{\prime}$ changes as well. We found no clusters in any of the data sets that supported a convincing association between $5^{\prime}$ changes and expression. Examining $5 \mathrm{~kb}$ regions shifted downstream of the TSS discovered more patterns than those upstream of the TSS, consistent with the existence of the $3^{\prime}$ pattern (Figure 5D) and absence of a $5^{\prime}$ pattern. From the cumulative evidence, we speculate that $\mathrm{CpG}$ island shore-like regions upstream of the TSS may not be independently associated with expression changes. Furthermore, while we observed that differential methylation patterns across the TSS were generally associated with gene silencing, $3^{\prime}$ methylation patterns correlated more often with downregulation than complete silencing (Supplementary Figure S12). $3^{\prime}$ hypermethylation events have previously been shown to 
A

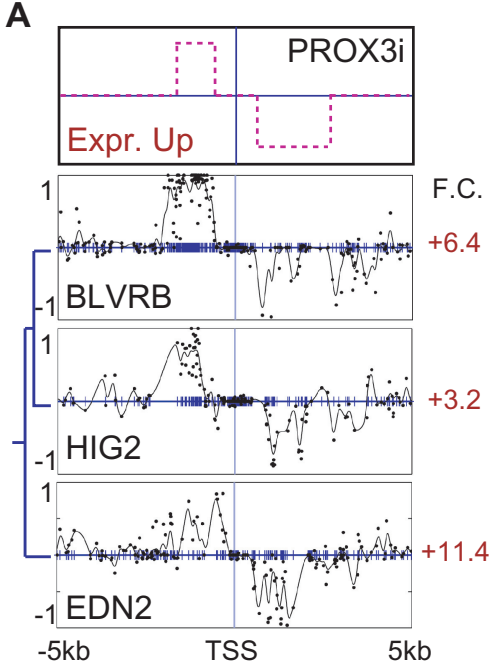

B

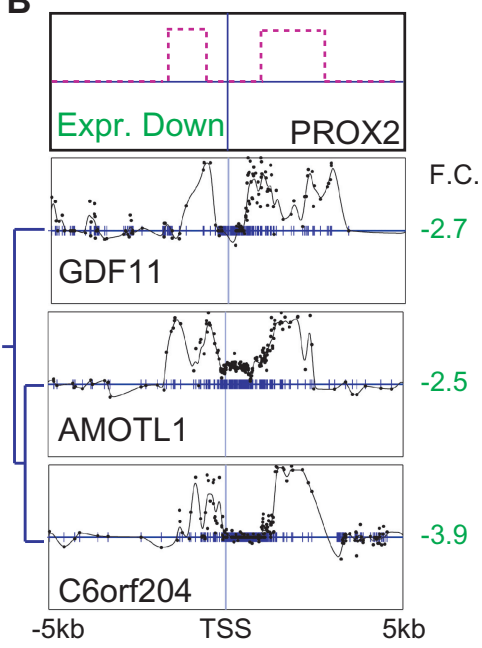

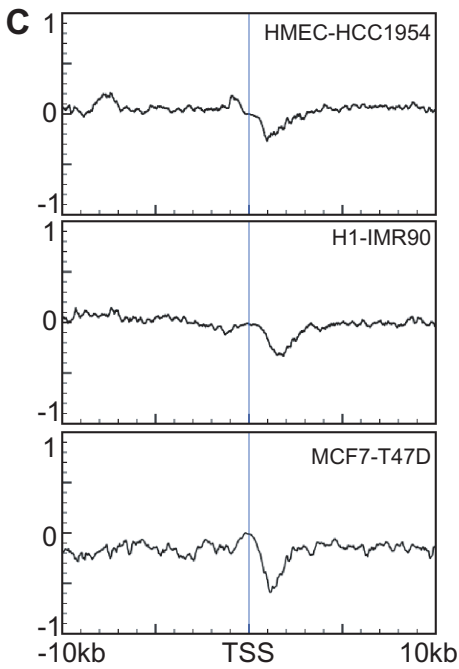

D

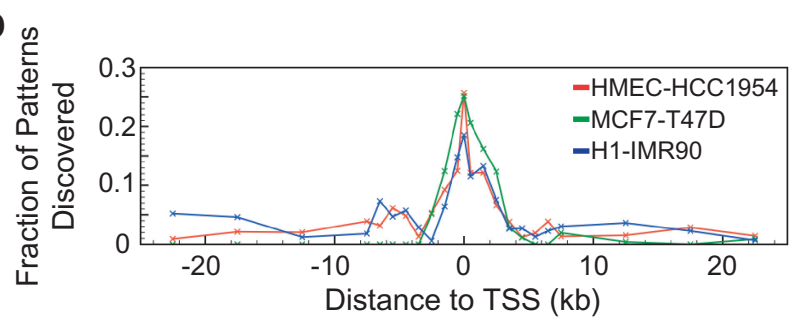

Figure 5. Patterns containing $3^{\prime}$ methylation changes are inversely correlated with expression, independent of changes $5^{\prime}$ of the TSS. (A, B) Top panel is a cartoon depicting the relevant pattern. Bottom panel shows an example subcluster from the default HMEC-HCC1954 clustering on a $10 \mathrm{~kb}$ region. Entire clusters for both parts are displayed in Supplementary Figure S11. (A) Subcluster exhibiting genes with a decrease in methylation on the $3^{\prime}$ side of the TSS and an increase in methylation $5^{\prime}$ of the TSS and with a significant increase in expression (PROX3i). (B) Subcluster exhibiting genes with an increase in methylation on both $3^{\prime}$ and $5^{\prime}$ sides of the TSS and with a significant decrease in expression (PROX2). (C) Meta-gene analysis of genes from significant clusters whose dominant pattern was identified as including a $3^{\prime}$-proximal component shows that the $3^{\prime}$ pattern is typically confined to within $3 \mathrm{~kb}$ of the TSS. From top to bottom, the three panels depict averages for HMEC-HCC1954, MCF7-T47D and H1IMR90 data sets. (D) The number of new genes identified increases downstream of the TSS, but not upstream. We started by identifying genes in significant clusters for a $5 \mathrm{~kb}$ region centered at the TSS. We iteratively moved the $5 \mathrm{~kb}$-wide region upstream of the TSS and identified all genes in significant clusters not found previously. This process was repeated for each of the region positions used by the gene list tool. The entire process was then repeated for downstream of the TSS.

affect expression (22), which supports the idea that the $3^{\prime}$ changes we observe could be potentially functional.

\section{Genomic features associated with particular DNA methylation patterns}

Gene ontology and expression signature analysis (23) of the genes found to have correlated differential methylation and expression showed that there was enrichment for cancer-related genes in HMEC-HCC1954 data, while there was enrichment for genes associated with differentiation in H1-IMR90 data. Long hypomethylated and partially methylated domains in cancer cells were previously observed to have distinct sequence contexts $(6,7)$. We find that genes with the TSS1 pattern are associated with a depletion of $\mathrm{CpG}$ density, Alu elements and gene density relative to all gene promoters (Figure $4 \mathrm{C}$ and $\mathrm{D}$ ). These depletions are observed in regions ranging from $10 \mathrm{~kb}$ up to $0.5 \mathrm{Mb}$ from the TSS. In summary, genes exhibiting the TSS1 pattern have the same distinct sequence properties as genes found in long hypomethylated domains (7).

There has been significant interest in addressing whether specific sequences direct or inhibit methylation at particular promoters or $\mathrm{CpG}$ islands $(5,24-26)$. One possibility is that different sequences may direct different methylation patterns. A preliminary search for motifs associated with each discovered pattern did not find a significant enrichment for any novel or known motifs associated with one pattern more than another. In addition, we find no significant association between each of the different observed methylation patterns and whether promoters are classified as CpG-rich or CpGpoor (Supplementary Figure S10).

\section{Quantifying the sensitivity of pattern discovery}

Because the true patterns of differential methylation that correlate with expression change are unknown, an obvious question is whether we have detected all correlative methylation patterns in the data set. To address this issue and quantify the method's ability to recover genes from known correlative patterns, we created a model to simulate differential methylation signatures and expression values. A complete description of the methods for simulated data is in the Supplemental Methods. 
Simulated genes with a predefined correlation between methylation and expression were added into two kinds of background data sets: randomly generated simulated genes with no correlation with expression (Supplementary Figure S13-S15) and real data. Using wholly simulated data we explored the types of patterns our method could discover. We successfully detected a variety of introduced patterns including peaks of differential methylation with fixed widths and locations relative to the TSS (Supplementary Figure S16), peaks with varying location (Supplementary Figure S17) and peaks with different variances in height (Supplementary Figure S18). More complex patterns were more easily detected, presumably because highly constrained curves are more likely to have similar Fréchet distances.

We also explored the method's ability to discover new patterns in a background of real methylation data. We introduced three simulated patterns (Supplementary Figure S19) into HMEC-HCC1954 WGBS data. We varied the number of genes added, and measured both the ability to discover the patterns and the number of genes correctly identified (Supplementary Figure S20). We found that the patterns were easily discoverable when at least 50-100 simulated genes were introduced, depending on the particular simulated pattern. Performance was impacted when one of the simulated patterns was too similar to the existing patterns (Supplementary Figure S21). Furthermore, we found that the fraction of simulated genes required to reliably detect a pattern decreased as the number of genes in the data set increased (Supplementary Figure S22). This suggests that one could detect rare patterns by simply concatenating multiple data sets.

\section{Enumerating genes with correlative methylation signatures}

Generating a list of genes for which expression and methylation changes are potentially linked is a primary interest of any genome-wide methylation profiling experiment. The method described above is tuned to discover patterns, not to create a gene list. Individual genes within good clusters will sometimes be false positives, and other genes will be excluded because their clusters do not meet the high purity threshold. To produce a better gene list, we executed our method on a set of overlapping $5 \mathrm{~kb}$ regions centered at a fixed set of locations around the TSS (Figure 6A). Comparison of gene lists from H1-IMR90 replicate WGBS data shows good concordance between replicates. To determine the extent to which genes are incorrectly included owing to the creation of errant clusters, we randomly scrambled the expression values in the HMEC-HCC1954 dendrogram. For 1000 such experiments using default clustering parameters, only seven experiments returned any false-positive genes: six reported a single false positive and a seventh returned two. We also tested the ability of the gene list method to recover introduced simulated genes (see 'Quantifying the sensitivity of pattern discovery' section above). For several simulated patterns, we found that the resultant gene lists included nearly all simulated genes when 50-100 were present (Supplementary Figure S20).

\section{Comparison to other approaches}

We compared the quality of the gene lists produced by our approach to lists constructed by two commonly used methods. For the DMR approach, regions of differential methylation are defined between two samples using a sliding window. DMRs are coupled to a particular gene using a cutoff for the distance between the DMR and the gene's TSS $(7,9)$. For the promoter-based approach, a fixed window around each gene's TSS defines the gene's promoter. If methylation changes substantially within this window, the gene is labeled as differentially methylated $(10,27)$. We optimized DMR- and promoter-based approaches for each data set using 69360 and 6174 parameter choices, respectively (Supplementary Figure S23 and Supplementary Table S3), while using a single common set of parameters for our approach across all data sets.

Judging the quality of the gene lists is difficult because there is no experimental gold standard data set for which the relationship between methylation at specific $\mathrm{CpG}$ sites and expression is well known for all genes. A correlation coefficient has often been used to quantify the association between methylation and expression. When applied to a list of genes for which methylation is predicted to associate with expression change, however, the correlation coefficient only judges one part of the method's performance. For example, by varying its parameters, the DMR method can produce short gene lists with strong correlations or long lists with weak correlations. To compare methods, we directly examine the trade-off between the total number of differentially expressed genes identified as potentially correlated versus the fraction of identified genes that are actually correlated in the predicted direction (Figure 6). This trade-off is somewhat analogous to comparing the rate of total positives to the rate of true positives, with the truenegative and false-negative rates being unknown. On the basis of these criteria, our approach clearly outperforms DMR- and promoter-based methods. For example, consider the HMEC-HCC1954 data (Figure 6B). When the DMR method is examined at a level where it correctly associates the direction of expression change $92 \%$ of the time, it returns only 26 genes $(0.7 \%$ of all differentially expressed genes). Our approach produces a list of 461 genes $(13 \%)$ at a correct association rate of $95 \%$. With less restrictive criteria, the DMR method gives a list of 717 genes $(20 \%)$ at a correct association rate of $71 \%$. Our technique gives a list of roughly the same size (761 genes) at a correct association rate of $93 \%$.

Table 1 presents results from each of the different data sets we analyzed and includes data from primary cells and cell lines. All analyses were performed using the default parameters. These results imply that prior approaches have underestimated the strength of the relationship between differential methylation and expression. With a better model of the underlying patterns of methylation change, it is clear that methylation and expression data are highly associated.

\section{Gene lists for low coverage data sets}

We next sought to examine how our approach performed with suboptimal data. Our technique returned similar 
A

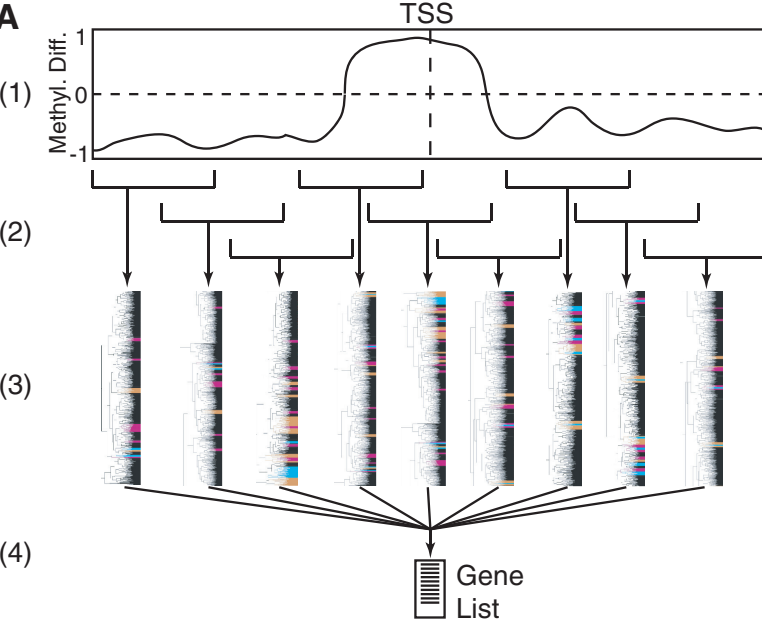

Compute Methylation

Signatures for Each Gene

Divide Signatures Using

Multiple Overlapping

Regions

Cluster Signatures for

Each Gene Within Each

Region; Identify

Significant Clusters

Select Genes Occurring in More Than $m$ Regions

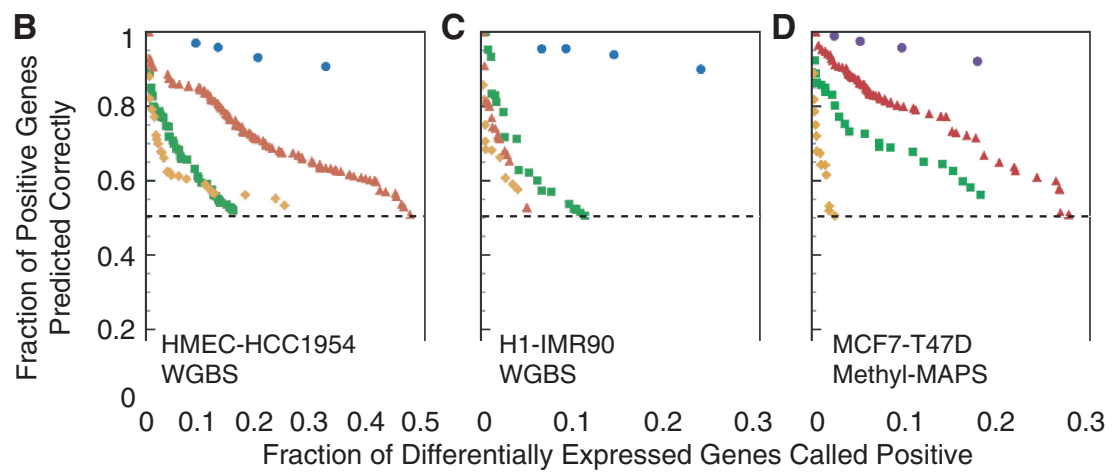

Our Method

DMR

Promoter, All CpGs

Promoter, Sig. CpGs

- - Random Guess

Figure 6. Gene lists generated by our method are of markedly greater length and quality than those generated by alternative methods. (A) Schematic of our process for generating gene lists. (B-D) Comparison of gene lists generated using our approach with those from optimized DMR and promoter-based methods for (B) HMEC-HCC1954 WGBS, (C) IMR90-H1 WGBS and (D) MCF7-T47D Methyl-MAPS data. The plot shows the trade-off between the number of genes identified as being associated with differential expression based on their methylation (x-axis) and the quality of the associations (y-axis), which is the fraction of identified genes for which the direction of expression change matches the expected direction based on methylation. Points up and to the right indicate better performance; $50 \%$ quality is equivalent to random guessing. Only optimal parameter choices with an inverse correlation between methylation and expression are shown for DMR- and promoter-based approaches (see Supplementary Figure S23 for further information about this optimization). Promoter-based approaches were optimized across both all CpG sites (All Sites) and all significant $\mathrm{CpG}$ sites (All Sig. Sites). The minimum number of required windows $m$ was varied in our method from 2 to 5 to show the effects of increased stringency.

Table 1. Summary of the numbers of genes identified by the gene list tool for each comparison

\begin{tabular}{|c|c|c|c|c|c|}
\hline Sample Comparison & $\begin{array}{l}\text { Differentially } \\
\text { expressed } \\
\text { genes }\end{array}$ & $\begin{array}{l}\text { CpGs with methylation } \\
\text { difference }>0.3\end{array}$ & $\begin{array}{l}\text { Genes with correct } \\
\text { association }\end{array}$ & $\begin{array}{l}\text { Genes with } \\
\text { incorrect } \\
\text { association }\end{array}$ & $\begin{array}{l}\text { Fraction of genes } \\
\text { with correct } \\
\text { association }\end{array}$ \\
\hline HMEC - НCC1954 & 3584 & 6979726 & 1150 & 118 & $91 \%$ \\
\hline MCF7 - T47D & 3740 & 1973564 & 664 & 57 & $92 \%$ \\
\hline ADS - ADS-Adipose & 2937 & 332489 & 138 & 15 & $90 \%$ \\
\hline ADS - ADS-iPSC & 3803 & 5917387 & 1124 & 93 & $92 \%$ \\
\hline H1 - H1-BMP4 & 4103 & 737003 & 65 & 3 & $96 \%$ \\
\hline H1 - H1-Mesendoderm & 2353 & 765051 & 59 & 5 & $92 \%$ \\
\hline H9-IMR90 & 5875 & 7669782 & 605 & 58 & $91 \%$ \\
\hline oocyte - ES cell (mouse) & 4727 & 1204883 & 334 & 25 & $93 \%$ \\
\hline sperm - ES cell (mouse) & 4580 & 4364748 & 1027 & 104 & $91 \%$ \\
\hline
\end{tabular}


results for the low coverage IMR90-H1 comparison, which has no minimum coverage cutoff, while DMRand promoter-based methods struggled (Figure 6C). We also ran experiments on the HMEC-HCC1954 sample pair to test the method's performance on downsampled data. First, we removed raw sequence reads at random, finding that WGBS data obtained with an average coverage as low as seven resulted in little loss in our method's ability to identify genes (Supplementary Figure S24A). We also removed methylation scores at random from the mapped data, and found that $94.5 \%$ of genes were still detected when $50 \%$ of the CpGs were removed (Supplementary Figure S24B). Additionally, our method outperforms the optimized DMR- and promoter-based methods for the MCF7-T47D sample pair collected using MethylMAPS, which contained $37.4 \%$ of the $\mathrm{CpG}$ sites from the $10 \mathrm{~kb}$ region centered at the TSS. These results suggest that our method is robust in the context of missing or low coverage data.

\section{DISCUSSION}

A summary of the patterns discovered in the data sets analyzed is presented in Figure 3. One primary advance of our approach is in its use of spatial information. Several of the patterns we found demonstrate the need for using spatial information about specific $\mathrm{CpG}$ sites when trying to connect differential methylation to expression change. For instance, the LONG0 pattern is positively correlated with expression, while the 3 '-hypomethylation pattern, commonly seen in conjunction with a $5^{\prime}$-hypomethylation event (PROX2i), is negatively correlated. Given methylation marks from one of these two cases, it is clear that the spatial information about specific $\mathrm{CpG}$ locations is necessary to successfully determine the direction of expression change. This may help explain an observation made during an analysis of 82 methylation data sets from human tissues and cell lines using reduced representation bisulfite sequencing (12). The authors observed that methylation changes in $\mathrm{CpG}$ island sites greater than $2 \mathrm{~kb}$ downstream were sometimes positively correlated with expression and sometimes negatively correlated. Based on our findings, one explanation for their observation is that they are observing a mixture of genes with LONG0 and $3^{\prime}$ patterns.

We also find that despite variation throughout the vicinity of a gene's promoter, expression change is often well correlated with only the methylation changes in a confined area relative to the TSS. This point is well supported by the many examples of the $3^{\prime}$-proximal pattern, which is seen with a variety of methylation activity upstream including hypermethylation, hypomethylation or little activity (Figure 3B). DMR-based methods are generally tuned to find wider regions than what we observe in our $3^{\prime}$-proximal clusters, to better identify genes with classical differential methylation at a $\mathrm{CpG}$ island promoter (e.g. TSS2 and TSS2i). While these methods can be tuned to locate narrower DMRs, this would result in more cases where contradictory DMRs exist near the TSS (e.g. one hypermethylated DMR and one hypomethylated DMR, such as PROX3 in Figure 3B). In these cases, there is no obvious way to decide which DMR may be influencing transcription without introducing pre-learned spatial information. As another example, the DMR approach has difficulty discriminating between the LONG0 pattern that is associated with a decrease in expression and the PROX1i and PROX2i patterns that are associated with an increase in expression. When set to find long regions downstream of the TSS, DMRs can be identified with positive correlation between expression and methylation. When set to find short regions, DMRs can be identified with negative correlations. However, no single set of DMR parameters can easily simultaneously capture each differential methylation pattern and its correlation with expression. These examples also underscore a fundamental limitation of the DMR method: it cannot be used to discover new patterns, but can only search for a limited set of relationships that are already known to exist.

Spatial information has proven useful to the analysis of other epigenetic data sets as well. Recently, a model considering the spatial locations of chromatin marks was used to train a support vector machine to predict chromatin signals at transcription factor binding sites (28). The authors divided each fixed region into $50 \mathrm{bp}$ bins, each of which was used as a feature in a vector for classification. The success of this approach demonstrates the potential of spatial models to better capture the nature of the epigenetic data than is possible with a simple windowbased approach. However, for analyzing DNA methylation, it is important to account for the relationship between such bins rather than treating them as independent features. For instance, in Figure 2C, the topmost and bottommost example curves have a similar-but slightly shifted-3'-proximal decrease in methylation and are both upregulated. If we used predefined nonoverlapping bins here, they would either be so narrow that the top peak and bottom peak lie in different bins, or so wide that the salient feature of the curve is diminished. Because our shape-similarity approach tolerates some movement of peaks in the x-direction, features such as these are naturally associated with one another.

Finally, our findings have implications for how DNA methylation should be assayed to preserve all potentially useful information. Using downsampled WGBS data and Methyl-MAPS data we demonstrated that we can discover the full set of patterns from Figure 3 without needing values at all $\mathrm{CpG}$ sites, as long as they are removed in a relatively unbiased manner (Supplementary Figure S24, Supplementary Data 3). However, information from some sites may be more informative than others. Many experimental methods attempt to assess a gene's methylation state by restricting analysis to only $\mathrm{CpG}$-rich regions, or to only a handful of $\mathrm{CpG}$ sites in and around the promoter. It is unclear whether such techniques measure methylation at the correct sites to discriminate the full spectrum of methylation patterns that correlate with transcriptional changes. As additional single-base resolution genome-wide DNA methylation data sets become available, we can explore which subsets of individual CpGs most inform promoter methylation patterns and thus 
need to be experimentally measured to accurately assess changes in a gene's methylation state.

\section{CONCLUSIONS}

Our findings suggest that characterizing gene promoters simply as 'methylated' or 'unmethylated' is insufficient. By considering the entire set of methylation changes near the promoter, we found and described a variety of methylation patterns that correlate with expression change. The power of our method is its ability to discover and separate distinct patterns without any prior knowledge about existing relationships, which cannot be accomplished with contemporary approaches. This allows us to use the full potential of unbiased genome-wide profiling of DNA methylation to reveal previously unknown information about methylation's functional role. Although we applied our method on regions of various widths around the TSS, all correlative patterns except those associated with the long hypomethylated domains were found within $5 \mathrm{~kb}$ of the TSS (Figure 5D). Interestingly, all patterns found in the cancer cell data sets were also found in the ES and iPSC cell data sets. While $5^{\prime}$ upstream patterns are observed, these appear to occur due to correlations with the $3^{\prime}$ downstream patterns. By appropriately capturing the diverse set of methylation patterns that exist, we observe a high level of association between changes in a gene's methylation state and changes in its expression.

A strength of the technique described here is its potential for expansion to examine more general epigenetic modifications. We have confined our analysis to data from genome-wide single-base resolution methods such as WGBS or Methyl-MAPS. However, similar approaches could be used to analyze the relationships between expression and other epigenetic patterns, such as 5-hydroxymethylcytosine, non- $\mathrm{CpG}$ methylation, and histone modifications. All expression data used in this study came from single-end short read RNA-Seq experiments. If long-read paired-end RNA-Seq data were available, it could easily be used with our method to understand alternate-promoter and isoform-specific methylation patterns. Considering the explosion of experiments aiming to profile epigenetic landscapes, this method represents a valuable tool for exploring the relationships between changes in epigenetic patterns and transcription both in normal cellular function and in human disease.

\section{SUPPLEMENTARY DATA}

Supplementary Data are available at NAR Online: Supplementary Tables 1-4, Supplementary Figures 1-24, Supplementary Methods, Supplementary Data 1-3 and Supplementary References [29-32].

\section{ACKNOWLEDGEMENTS}

The authors thank Yu-Tseh Chi and Tao Ju for helpful discussions about curve comparison algorithms. The authors also thank Christopher Schlosberg for helpful discussions and reading of this manuscript.

\section{FUNDING}

National Institutes of Health [NIH R00 CA127360, NIH R21 LM011199 to J.R.E.]; the Department of Defense to J.R.E.]; a Siteman Cancer Center Breast Cancer Program career development award [to J.R.E.]. Funding for open access charge: The Internal Fund from the Department of Medicine, Washington University in St. Louis.

Conflict of interest statement. None declared.

\section{REFERENCES}

1. Laurent,L., Wong,E., Li,G., Huynh,T., Tsirigos,A., Ong,C.T., Low,H.M., Kin Sung,K.W., Rigoutsos,I., Loring,J. et al. (2010) Dynamic changes in the human methylome during differentiation. Genome Res., 20, 320-331.

2. Ehrlich,M. (2002) DNA methylation in cancer: too much, but also too little. Oncogene, 21, 5400-5413.

3. Rakyan,V.K., Down,T.A., Balding,D.J. and Beck,S. (2011) Epigenome-wide association studies for common human diseases. Nat. Rev. Genet., 12, 529-541.

4. Saxonov,S., Berg,P. and Brutlag,D.L. (2006) A genome-wide analysis of $\mathrm{CpG}$ dinucleotides in the human genome distinguishes two distinct classes of promoters. Proc. Natl Acad. Sci. USA, 103, 1412-1417.

5. Doi,A., Park,I.H., Wen,B., Murakami,P., Aryee,M.J., Irizarry,R., Herb,B., Ladd-Acosta,C., Rho,J., Loewer,S. et al. (2009) Differential methylation of tissue- and cancer-specific $\mathrm{CpG}$ island shores distinguishes human induced pluripotent stem cells, embryonic stem cells and fibroblasts. Nat. Genet., 41, 1350-1353.

6. Hon,G.C., Hawkins, R.D., Caballero,O.L., Lo,C., Lister,R., Pelizzola,M., Valsesia,A., Ye,Z., Kuan,S., Edsall,L.E. et al. (2012) Global DNA hypomethylation coupled to repressive chromatin domain formation and gene silencing in breast cancer. Genome Res., 22, 246-258.

7. Hansen,K.D., Timp,W., Bravo,H.C., Sabunciyan,S., Langmead,B., McDonald,O.G., Wen,B., Wu,H., Liu,Y., Diep,D. et al. (2011) Increased methylation variation in epigenetic domains across cancer types. Nat. Genet., 43, 768-775.

8. Berman,B.P., Weisenberger,D.J., Aman,J.F., Hinoue,T., Ramjan,Z., Liu,Y., Noushmehr,H., Lange,C.P., van Dijk,C.M., Tollenaar,R.A. et al. (2012) Regions of focal DNA hypermethylation and long-range hypomethylation in colorectal cancer coincide with nuclear lamina-associated domains. Nat. Genet., 44, 40-46.

9. Lister,R., Pelizzola,M., Dowen,R.H., Hawkins,R.D., Hon,G., Tonti-Filippini,J., Nery,J.R., Lee,L., Ye,Z., Ngo,Q.M. et al. (2009) Human DNA methylomes at base resolution show widespread epigenomic differences. Nature, 462, 315-322.

10. Edwards,J.R., O'Donnell,A.H., Rollins,R.A., Peckham,H.E., Lee,C., Milekic,M.H., Chanrion,B., Fu,Y., Su,T., Hibshoosh,H. et al. (2010) Chromatin and sequence features that define the fine and gross structure of genomic methylation patterns. Genome Res., 20, 972-980.

11. Bock,C. (2012) Analysing and interpreting DNA methylation data. Nat. Rev. Genet., 13, 705-719.

12. Varley,K.E., Gertz,J., Bowling,K.M., Parker,S.L., Reddy,T.E., Pauli-Behn,F., Cross,M.K., Williams,B.A., Stamatoyannopoulos,J.A., Crawford,G.E. et al. (2013) Dynamic DNA methylation across diverse human cell lines and tissues. Genome Res., 23, 555-567.

13. Eckhardt,F., Lewin,J., Cortese,R., Rakyan,V.K., Attwood,J., Burger,M., Burton,J., Cox,T.V., Davies,R., Down,T.A. et al. (2006) DNA methylation profiling of human chromosomes 6, 20 and 22. Nat. Genet., 38, 1378-1385. 
14. Hansen,K.D., Langmead,B. and Irizarry,R.A. (2012) BSmooth: from whole genome bisulfite sequencing reads to differentially methylated regions. Genome Biol., 13, R83.

15. Alt,H. and Godau,M. (1995) Computing the Frechet distance between two polygonal curves. Int. J. Comput. Geom. Appl., 5, $75-91$.

16. Eiter,T. and Mannila,H. (1994) Computing discrete Fréchet distance. Tech. Report CS-TR-2008-0010. University of Texas at San Antonio, San Antonio, TX.

17. Aronov,B., Har-Peled,S., Knauer,C., Wang,Y. and Wenk,C. (2006) In: Proceedings of the 14th conference on Annual European Symposium - Volume 14. Springer-Verlag, Zurich, Switzerland, pp. 52-63.

18. Rada-Iglesias,A., Bajpai,R., Swigut,T., Brugmann,S.A., Flynn,R.A. and Wysocka,J. (2011) A unique chromatin signature uncovers early developmental enhancers in humans. Nature, 470, 279-283.

19. Lister,R., Pelizzola,M., Kida,Y.S., Hawkins,R.D., Nery,J.R., Hon,G., Antosiewicz-Bourget,J., O'Malley,R., Castanon,R., Klugman,S. et al. (2011) Hotspots of aberrant epigenomic reprogramming in human induced pluripotent stem cells. Nature, 471, 68-73.

20. Kobayashi,H., Sakurai,T., Imai,M., Takahashi,N., Fukuda,A., Yayoi,O., Sato,S., Nakabayashi,K., Hata,K., Sotomaru,Y. et al. (2012) Contribution of intragenic DNA methylation in mouse gametic DNA methylomes to establish oocyte-specific heritable marks. PLoS Genet., 8, e1002440.

21. Majewski,J. and Ott,J. (2002) Distribution and characterization of regulatory elements in the human genome. Genome Res., 12, $1827-1836$

22. Appanah,R., Dickerson,D.R., Goyal,P., Groudine,M. and Lorincz,M.C. (2007) An unmethylated 3' promoter-proximal region is required for efficient transcription initiation. PLoS Genet., 3, e27.

23. Culhane,A.C., Schroder,M.S., Sultana,R., Picard,S.C., Martinelli,E.N., Kelly,C., Haibe-Kains,B., Kapushesky,M.,
St Pierre,A.A., Flahive,W. et al. (2012) GeneSigDB: a manually curated database and resource for analysis of gene expression signatures. Nucleic Acids Res., 40, D1060-D1066.

24. Das,R., Dimitrova,N., Xuan,Z., Rollins,R.A., Haghighi,F., Edwards,J.R., Ju,J., Bestor,T.H. and Zhang,M.Q. (2006) Computational prediction of methylation status in human genomic sequences. Proc. Natl Acad. Sci. USA, 103, 10713-10716.

25. Bock,C., Paulsen,M., Tierling,S., Mikeska,T., Lengauer,T. and Walter,J. (2006) CpG island methylation in human lymphocytes is highly correlated with DNA sequence, repeats, and predicted DNA structure. PLoS Genet., 2, e26.

26. Feltus,F.A., Lee,E.K., Costello,J.F., Plass,C. and Vertino,P.M. (2003) Predicting aberrant $\mathrm{CpG}$ island methylation. Proc. Natl Acad. Sci. USA, 100, 12253-12258.

27. Meissner,A., Mikkelsen,T.S., Gu,H., Wernig,M., Hanna,J., Sivachenko,A., Zhang,X., Bernstein,B.E., Nusbaum,C., Jaffe,D.B. et al. (2008) Genome-scale DNA methylation maps of pluripotent and differentiated cells. Nature, 454, 766-770.

28. Arvey,A., Agius,P., Noble,W.S. and Leslie,C. (2012) Sequence and chromatin determinants of cell-type-specific transcription factor binding. Genome Res., 22, 1723-1734.

29. Rollins,R.A., Haghighi,F., Edwards,J.R., Das,R., Zhang,M.Q., $\mathrm{Ju}, \mathrm{J}$. and Bestor,T.H. (2006) Large-scale structure of genomic methylation patterns. Genome Res., 16, 157-163.

30. Trapnell,C., Pachter,L. and Salzberg,S.L. (2009) TopHat: discovering splice junctions with RNA-Seq. Bioinformatics, 25, 1105-1111.

31. Robinson,M.D., McCarthy,D.J. and Smyth,G.K. (2010) edgeR: a Bioconductor package for differential expression analysis of digital gene expression data. Bioinformatics, 26, 139-140.

32. Robinson,M.D. and Oshlack,A. (2010) A scaling normalization method for differential expression analysis of RNA-seq data. Genome Biol., 11, R25. 